\title{
A Constructivist View of Newton's Mechanics
}

\author{
H. G. Solari ${ }^{1}$ (D) M. A. Natiello ${ }^{2}$ (D) \\ Published online: 4 December 2018 \\ (C) The Author(s) 2018
}

\begin{abstract}
In the present essay we attempt to reconstruct Newtonian mechanics under the guidance of logical principles and of a constructive approach related to the genetic epistemology of Piaget and García (Psychogenesis and the history of science, Columbia University Press, New York, 1989). Instead of addressing Newton's equations as a set of axioms, ultimately given by the revelation of a prodigious mind, we search for the fundamental knowledge, beliefs and provisional assumptions that can produce classical mechanics. We start by developing our main tool: the no arbitrariness principle, that we present in a form that is apt for a mathematical theory as classical mechanics. Subsequently, we introduce the presence of the observer, analysing then the relation objective-subjective and seeking objectivity going across subjectivity. We take special care of establishing the precedence among all contributions to mechanics, something that can be better appreciated by considering the consequences of removing them: (a) the consequence of renouncing logic and the laws of understanding is not being able to understand the world, (b) renouncing the early elaborations of primary concepts such as time and space leads to a dissociation between everyday life and physics, the latter becoming entirely pragmatic and justified a-posteriori (because it is convenient), (c) changing our temporary beliefs has no real cost other than effort. Finally, we exemplify the present approach by reconsidering the constancy of the velocity of light. It is shown that it is a result of Newtonian mechanics, rather than being in contradiction with it. We also indicate the hidden assumption that leads to the (apparent) contradiction.
\end{abstract}

Keywords Newtonian mechanics $\cdot$ No arbitrariness principle $\cdot$ Objective versus subjective description - Law of inertia · Central forces · Energy conservation · Laws of nature · Genetic epistemology

\section{Introduction}

Probably every physics student has wondered what is behind Newton's laws. Some may even have read the Principia (Newton 1687) looking for an answer, just to find a few indications in the introduction followed by a large hiatus taking them directly to Newton's

M. A. Natiello

mario.natiello@math.lth.se

1 Departamento de Física, FCEN-UBA and IFIBA-CONICET, Buenos Aires, Argentina

2 Centre for Mathematical Sciences, Lund University, Lund, Sweden 
axioms, with no clue on how to produce them. While some everyday experiences can be used to grasp the first and third law, the second law appears to be arbitrary (to say the least). Are there principles operating behind the laws? Is there a unity among them? What do they hide? These questions have bugged all scientists exposed to physics as they have bugged us since we were students. They are relevant for the constructivist approach known as second order science (Mueller 2014; Lissack 2017) as well. Are the foundations of physics intuitions of powerful minds subject to no other rule than an a posteriori test of usefulness? Are they useful pragmatic beliefs subject to no rule of construction [an idea going back at least to Peirce (Hartshorne and Weiss 1931; Burks 1946)] to be replaced only when a new genius handles us a new set of axioms? Is then reasoning in physics restricted to the deductive form, to instrumental reason? Or is there room for critical reason searching for the foundations? Shouldn't a philosophy of physics follow Hegel's dictum: "progress in philosophy is rather a retrogression and a grounding or establishing by means of which we first obtain the result that what we began with is not something merely arbitrarily assumed but is in fact the truth, and also the primary truth." (Hegel 2001, par.101)?

On the utilitarian side, reasoning is the most powerful adaptation of the animal we call human being to the conditions of Nature, for reason allows humans to foresee the outcomes of events and to act taking advantage of opportunities, avoiding dangers and unfavourable conditions. As a consequence, inasmuch as reasoning enhances adaptive capabilities, it is a part of natural selection. This selection took place in front of the difficulties placed by natural phenomena.

On the idealistic side, a long-term desire of mankind is to understand Nature. What is implied in it? Understanding Nature is a search for harmony, it means trying to see how all apparent contingencies (good or bad) are in fact aspects of different expressions of the totality implied in Nature (or can be constructed as such). Even in the quest for understanding elusive matters as mathematical chaos, the intellectual exercise is not an apology of what exists beyond all possible organisation/understanding but rather, to find the perspective that allows us to see harmony in what, at first sight, was perceived as disorder (Solari et al. 1996). This view is related to the concept of praxis, which in phenomenology is considered "a human activity that is pursued as an end in itself, that is, a terminal goal of human living rather than as practical problem-solving (techne)" (Heelan and Tymieniecka 2003).

Understanding Nature demands the development of several processes, being the most immediate one to strip off the particularities that dress observations (in relation to the matter under discussion), thus disclosing properties common to them and relations that often have been named the essence (Husserl 1983). This process is associated to abstraction. We emphasise here that in these terms, essence is not absolute but instead relative to the posed questions.

For example, for any discussion of the biomechanics of a horse, the colour of its coat is irrelevant, yet if what we are to discuss is the "market price" of the horse, the coat usually plays a significant role in a predictive theory. The reader may want to distinguish the act of abstracting away colour when considering biomechanics from more demanding idealisations requiring some sort of extrapolation such as e.g., regarding the dynamics of frictionless bodies as the (unattainable) limit of the behaviour of bodies subject to different degrees of friction. For this process Galileo used the word idealisation [see e.g., Discorsi e Dimostrazioni Matematiche Intorno a Due Nuove Scienze (Galilei 1914, day 4)]. We will use in the sequel the word idealisation without distinguishing between both types of processes (we notice that Husserl used the word ideation for the process of intuition transforming the observed into ideas). In this process of idealisation experience is put in terms 
of relations between idealised (abstract) objects through synthetic judgements (Kant). These relations are tentative and are always to be suspected of being in contradiction with new observations; we call them theories.

Descartes introduced the expression "laws of Nature" referring to principles of physics concerning the motion of bodies [Discourse on method (Descartes 2015, p. 19)]. The dogma that Nature follows its own laws, which can then be discovered, soon gave birth to a new child: Newton's laws, presented in his Philosophiæe Naturalis Principia Mathematica, (Newton 1687). These developments represent the beginning of the scientific revolution which includes the mathematisation of the idealised relations (Galileo) and a reconstruction of the idea of "cause" to be compatible with idealised relations. Yet, Aristotle's dictum "we think we do not have knowledge of a thing until we have grasped its why, that is to say, its cause" (Aristotle, 1994-2010) remained valid. In this sense, the quest of knowledge maintained a continuity from the ancient greeks to the modern scientists.

The understanding of causative relations is the long-term programme of science. However, the relation of cause and effect is not free of debate. It was tackled by Hume (1896) as a necessary relation between contiguous objects. For Hume, causes must always precede effects. As realised later by Russell (1912-1913), cause became a confusing term seldom used by scientists. He indicated that instead of causes scientists use "state functional relations between certain events at earlier and later times or at the same time", he uses the name determinants for these events. Both the determinants and the relations are necessary to answer Aristotle's question: why it happens? and as such, they are Aristotelian causes.

In the crusade for the "elimination of metaphysics", Carnap (1959) asserts "we say of a thing or process $y$ that it 'arises out of' $x$ when we observe that things or processes of kind $x$ are frequently or invariably followed by things or processes of kind $y$ (causal connection in the sense of a lawful succession)." Cause in Carnap takes the form of a relation between observed things or processes, insisting in Hume's old empiricist view. Let us challenge such extreme empiricist notion. Consider a rectangular cloth suspended by strings tied to its four corners. If we let a ball rest on the cloth we observe a deformation of the cloth and the formation of some wrinkles that evidence tensions. Is the ball the cause of the wrinkles and the deformation? Or perhaps the wrinkles have attracted the ball? Such a simple matter is not easy to decide using Carnap's dictum. The deformation and the presence of the ball resting on the cloth are simultaneous; there is no precedence in time. However, it has never been observed that by making wrinkles on a cloth we can attract balls in the surroundings. Clearly, we say that the ball is the cause of the deformation and the wrinkles [this example is adapted from Kant (1787, p. 183)]. Thus, causes can very well be simultaneous with effects and the decision about what is the cause of a phenomenon is taken based on reason and on other experiences. But physics requires more than this type of analysis. Take the ball, lift it and let it drop. Is there a relation between what makes the ball fall and the cloth to wrinkle? We all know that there is a relation between not-falling (staying on the cloth) and wrinkles, and both events point towards an element left outside in the previous analysis: gravitation. Without gravitation (or with lesser gravitation) we would observe no (or smaller) deformation, and no (or slower) fall. Gravitation can be inferred but not observed (the same can be said of tension). In our observations, gravitation is acting as a necessary element in our explanation, i.e., a cause in Aristotle's perception. Thus, if we want to construct knowledge we have to abandon the crusade against metaphysics and allow ingredients that are produced by our mind; in this case elements such as gravitation that are not sensible but rather inferred (call them meta-sensible if it pleases the reader). We shall call them metaphysical (where does physics end?). We notice that by so doing the time-order between causes and effects is erased to a good extent, for gravitation is there before, during 
and after the time the ball rests on the cloth, although what it counts for us is that it was before and during the experiment. The fall of the ball on the cloth (with the subsequent coming to rest) and the wrinkles are the result of at least one common cause. In the empiricist view, metaphysics is not absent but rather hidden in habits and commonplaces. We cannot account for experience without some degree of idealisation, i.e., metaphysics. We observe that putting metaphysics back into science is suggested as well in Lissack (2017).

Our use of cause will indicate relations of the kind described by Russell unless otherwise stated. For example, we will later state that "force is the cause of acceleration" since force expresses a functional relation between a change in velocity, time and other physical characteristics. Notice that in this case there are no determinants.

Equipped with reasoning, idealisation and an understanding of cause in order to address natural phenomena, in the present work we will try to distinguish the diverse elements that contribute to the construction of classical (Newtonian) mechanics elaborating from the construction of reality in childhood as described by genetic epistemology. We find three clear sources for these contributions, namely: (a) requisites of reason, named principles (b) intuitive building blocks that are constitutive of our understanding after their production in our early contact with the world (called axioms) and (3) provisional assumptions with respect to the organisation of the sensible world which, as pragmatical beliefs (Hartshorne and Weiss 1931), are the main target of procedures such as falsation (or falsification) (Popper 1959) and retroduction (often called abduction) (Hartshorne and Weiss 1931; Burks 1946).

With the word principle we mean a requisite of reason prior to any theorisation or empiric tests. Two principles will be stated in the next section, being the Principle of no Arbitrariness (NAP) the most far-reaching. We note on passing that the word "principle" has other uses as well (e.g., Newton's third law is also called the Principle of Action and Reaction). As for axioms, they are broad building blocks that constitute the basis of a theory. In theories about Nature, axioms are to a large extent motivated by the way human beings relate to Nature and may be questioned if they fail in this respect. Axioms are related to intuitions in Husserl's phenomenology (Husserl 1983), since we proceed first by removing spatio-temporal determinants and further incorporating the concept (the essence, Eidos). Husserl states "seeing an essence is precisely intuition". Within a mathematical theory, however, the axioms are accepted without need for proof. Assumptions, in turn, are local decisions in order to limit the scope of the theory according to the desired goals. Consider for example: "in the sequel we consider forces of instantaneous action at a distance". The axioms and previous theory may encompass other types of forces as well, but our local interest in a given context is delimited by making this (or other) assumption. Mathematical theories of Nature are usually built up by axioms and assumptions, where axioms represent our intuition of Nature, our experience, and are the starting point of empiricist theories. We add here a higher level to this structure which comes from the subject and it precedes and rules intuitions. We argue that this principle is a fundamental element in Physics.

This work is the result of an interplay between philosophy, psychology, mathematics and physics, traditions of thoughts that we do not conceive as autonomous but rather different realisations of the same general form that we call reason, this is, reason as it is needed in this or that field of use (application). The reader not familiar with this synthetic form, and perhaps not comfortable in all of the particular forms is invited to skip the unfriendly details in a first reading. For example, proofs are unavoidable for mathematicians but physicists will often adopt a "for the moment I trust you" attitude in a first reading.

The mathematisation of NAP is in Sect. 2, the application of NAP to space and time and a new insight into Galileo's transformations is to be found in Sect. 3. Sections 4 and 5 
show how NAP enters in Newton's laws. These two sections contain the most demanding mathematics. Section 6 is devoted to reflecting about Newton's mechanics with connections to other criticisms and analyses of Newton's work. Section 7 treats the speed of light within the Newtonian system. We first show that what is measured is expected to be independent of the Galilean frame. Next we uncover a hidden assumption that leads to the wellknown problem between a constant speed of light, Newton's law and the hidden assumption. Readers mainly interested in this matter may want to skip Sects. 4 and 5 (Sect. 3 is required). Within this discussion the last mathematical difficulty appears in Sect. 7.1.2 where we adopt, for the sake of the exercise, absolute time and absolute space showing that even substantivalists (Maudlin 1993) have options within the Newtonian world to account for a constant speed of light. This is an example of how constructivism offers new forms of thinking physical problems. Finally Sect. 8 sums up the manuscript.

Natives of psychology and philosophy will have to endure our speaking of those subjects as foreigners. Further, we do not pretend to be disciplinary experts in all of the above mentioned fields, rather we use the disciplined thought to corroborate, support and sharpen our own (free) thinking. The reader is warned that this work is made of undisciplined thinking more in the "prophetic tradition" than in the "scholastic" one (Nisbet 1971).

\section{No Arbitrariness Principle}

In any rational construction attempting to logically articulate the natural world, we are bound to keep elements not based in reason or evidence outside the construction if possible. Since what is determined by chance, whim, or impulse, and not by necessity, reason, or principle is called arbitrary, ${ }^{1}$ we adopt the attitude of rejecting arbitrary practices. If it were not possible to achieve a full exclusion of arbitrariness, we must make sure that the arbitrariness introduced plays no role in answering valid questions with respect to Nature. While it is certainly possible to uproot the questions whose answers depend on arbitrary decisions, in constructing a theory we must strive to avoid the arbitrariness as much as possible and to keep the valid questions as broad as possible.

This approach to understanding will be called the No Arbitrariness Principle. Resigning this principle i.e., admitting arbitrariness as a basic ingredient, would render communication extremely difficult. The principle delimits the conditions with which knowledge about Nature is to develop. As such, it is therefore prior to physics and experience and cannot be questioned empirically. In a theory of natural phenomena, subsequent axioms and even more assumptions are connected to empiric evidence and may be falsified empirically, thus suggesting the need for a more adequate theory.

Struggling to avoid arbitrariness in a physical description immediately reflects in the invariance of this description with respect to elections for which we do not have a reasonable foundation. For example, measurements imply comparisons and the units of measurement are arbitrary to a good extent, yet velocity will always be written in terms of a ratio between distance and time. This form is independent of arbitrary choices of units, although the figure of the velocity changes according to the chosen units.

Within the mathematical theory we are about to develop, we will formulate these ideas as principles:

\footnotetext{
${ }^{1}$ See for example, The Free Dictionary, https://www.thefreedictionary.com/.
} 
Principle 1 There is a material world we perceive with our senses (including experiments).

We call this world Nature and we strive to find laws that describe its perceived organisation. In this context we state the main idea of this work:

Principle 2 [No Arbitrariness Principle (NAP)] No knowledge of Nature depends on arbitrary decisions.

In short, NAP establishes that: If there is no reason for it, we shall make no difference. In this simple form it can be recognised as Leibniz' principle of sufficient reason (Ballard 1960) put in a cognitive context. ${ }^{2}$

The arbitrary decisions adopted while constructing a theory can be viewed as forming a group of transformations operating over the laws and/or the magnitudes involved in the theory. Each choice of elements in the set of arbitrary assumptions is used to produce a presentation of the theory and all presentations must be equivalent, i.e., conjugated by elements of the group of arbitrary decisions (we will formalise this idea in mathematical terms in Sect. 2.2).

It is important to notice that this approach evolves from the construction of reality by the child. We quote Piaget on groups: "There is a mutual dependence between group and object; the permanence of objects presupposes elaboration of the group of their displacements and vice-versa. On the other hand, everything justifies us in centering our description of the genesis of space around that of the concept of group. Geometrically, ever since $\mathrm{H}$. Poincaré this concept has appeared as a prime essential to the interpretation of displacements [...] But it is necessary to remember that we shall attribute the widest meaning to this concept for if, as recent works have shown, the logical definition of the group is inexhaustible and involves the most essential processes of thought, it is possible, purely from our psychological point of view, to consider as a group every system of operations capable of permitting a return to the point of departure." (Piaget 1999)

\subsection{Objectivity and Intersubjectivity}

If we observe a cat stalking a bird in our garden, we perceive a distance between cat and bird and we have indications that both cat and bird perceive something similar, since the cat does not launch the attack until the distance is short enough and the bird does not fly away until the cat is threatening close.

\footnotetext{
${ }^{2}$ The principle of sufficient reason (PSR) has made little or no progress since the Clarke-Leibniz polemic (Ballard 1960). A discussion among substantivalists and relationalists has re-emerged with Relativity Theory but in a form in which philosophy is subordinated to the "success" of physics. Within such an intellectual disposition it has been declared that "neither the PSR nor the PII enjoys at present unquestionable philosophical credentials" (Maudlin 1993) (PII stands for Principle of Identity of Indiscernibles). Actually, the discussion appears as frozen in time, lacking critical contributions and it still revolves around God. In contrast, NAP stands for a critical as well as operative view, it has a long tradition in mathematics where the expression "without lost of generality we can assume..." is frequent in proofs, meaning that the results will not depend on our arbitrary choices but for the sake of the argument a choice must be made. This is: arbitrariness should not and will not bear any consequence in what is being proved. The metacognitive instruction received by every student of physics: "the final result should not depend in your election of units or your choice of path to reach it" manifests the same conviction: arbitrariness has no part in truth.
} 
In the next Section we will formalise these perceptions in the concept of relative position, saying that it is objective (as well as cat and bird are), while our perceptions of it are intersubjective, meaning that the cat's, bird's and any observer's perceptions can be put in one-to-one correspondence. In most of the text we will make no difference between objective and intersubjective. When we make a difference it corresponds to the fact that for sensible objects we produce the internal representation by experience or intuition (eidetic seeing or ideation in Husserl) leaving aside contingent elements, but there are other objects (essence, Eidos) which are the datum of eidetic intuition (Husserl 1983). Thus we call 'objective' representations produced by ideation and 'intersubjective' all representations irrespective of the origin. As such, space is objective but time is only intersubjective. A corresponding distinction was made by (Kant 1787) in his discussion of "the a priori of knowledge".

The internal representations need not be the same but just corresponding ones. Let $x_{C B}$ be a symbol for the idea of relative position between cat and bird. We are used to think that the observer $a$ represents it as $x_{C B}=x_{C}^{a}-x_{B}^{a}$, which is in fact a synthetic judgement-not a mathematical one-since it proposes a relation between three different concepts: the positions $x_{C}^{a}, x_{B}^{a}$ of cat and bird relative to the observer (or to a reference of her/his choice) and the relative position between cat and bird $x_{C B}$. Since the observer entered the picture by the arbitrary action of referring positions with respect to her/himself, this expression must be subject to the no arbitrariness principle, NAP. It is important to realise that $x_{C B}$ is a symbol that relates to the sensory perceived world, not an internal mathematical object.

The relation between mathematics and objectivity has been addressed from several points of view in the past. For example: the objectivity of mathematics (post-Hilbert), the objectivity of statistical models (Rasch 1968), or the problem of meaning and objectivity (Hill and Haddock 2000). None of the points of view which we are aware of attempts to formalise objectivity in mathematical terms.

\subsection{Mathematisation of Objectivity}

Consider a set $E$ of concepts/magnitudes and a set $A$ of arbitrary decisions, necessary for its representation. Let $R_{a}(e)$ be a representation of the concept $e \in E$ depending on the arbitrary decision $a \in A$ and let $F$ be a "natural law" expressed as $F\left(R_{a}(e)\right)=0$. For example, if the concept $e$ is "distance", $R_{a}(e)$ is the real number giving the distance according to $a$ and the "natural law" is some mathematical expression involving this number, such as $R_{a}(e)-C=0$ if the natural law expresses that this distance has the constant value $C$.

Definition 1 (Objectivity) A law is objective if $F\left(R_{a}(e)\right)=0$ holds for any $a \in A$.

Consider an invertible transformation $T_{a b}$ that maps a representation $R_{a}$ onto another representation $R_{b}\left(T_{a b} \circ T_{b a}=I d\right)$. By NAP, Definition 1 can be restated as: 


$$
F\left(R_{a}(e)\right)=0 \Leftrightarrow F\left(T_{b a} R_{a}(e)\right)=F\left(R_{b}(e)\right)=0 .
$$

The transformations $T_{a b}$ form a group ${ }^{3} T$. We assume that:

a) $T_{a b} \circ T_{b c}=T_{a c}$, there exists a composition law.

b) $T_{a a}=I d$, there exists an identity.

c) $T_{a b}=\left(T_{b a}\right)^{-1}$ or $\left(T_{b a} \circ T_{a b}\right)=I d$, an inverse exists.

d) $T_{a b} \circ\left(T_{b c} \circ T_{c d}\right)=\left(T_{a b} \circ T_{b c}\right) \circ T_{c d}$, the composition law is associative.

As a consequence, $T_{a b} \circ\left(T_{b c} \circ T_{c a}\right)=I d=\left(T_{a b} \circ T_{b c}\right) \circ T_{c a}$ and $T_{a b} \circ\left(T_{b c} \circ T_{c d}\right)=T_{a b} \circ T_{b d}=$ $T_{a d}=T_{a c} \circ T_{c d}=\left(T_{a b} \circ T_{b c}\right) \circ T_{c d}$.

\section{Space, Time and Observers}

The system of concepts that allows the organisation of reality is dialectical, with multiple concepts coming into existence at once. Certain concepts in this system are meaningful only in relation to other concepts introduced simultaneously. In all dialectical constructions, the tension of the opposites is the engine of understanding, but the terms in opposition present no difference between them other than being just the terms of the opposition. Hegel's dialectic of being and not-being is perhaps the clearest example of this constructive procedure (Hegel 2001). In our case, the concepts: ego, alter, identity, object, space and time are the fundamental building blocks for the construction of understanding; they cannot be referred to previous concepts because understanding can only be referred to its opposite: not-understanding. Thus, any attempt at defining these terms will never be completely satisfactory since we will have to resort to some complicity from the intuitions of the reader. We call this process dialectical openings to understanding. ${ }^{4}$ In practical terms, when we mention a concept (in this section) using a word that is defined later in the text,

\footnotetext{
3 We notice that in a structural realist work (Delhôtel 2017) has considered the restricted case of transformations concerning space-time following the relativistic tradition born out of Poincaré's principle. He identifies "a major item on the structural realist agenda: being able to demonstrate that structural features are precisely and only those that are preserved across theory change, in one fashion or another, exactly or approximately" and later identifying "equivalence principles" as one of such structures. What we discuss here is stronger and deeper, because we account for the reasons why equivalence structures are required in a more general setting than Poincaré's Relativity Principle (that will be briefly and critically discussed below). The structure identified in Delhôtel (2017) is a particular case of the mathematisation of NAP presented here. The requirement of having a group of transformations connecting descriptions differing in arbitrary choices is recognised in Delhôtel (2017, sec. 3) not as a requisite of reasoning but only as a consequence of the postulates of the dynamics.

4 In our daily quest to understand nature we make use of ideas and concepts previously produced by our society that we have naturalised (included in Nature although they belong to our cultural baggage). However, the recursion to previous understanding has an end and it is not always possible. When we arrive to such a dead end while exercising the critical reason in the search for the fundamentals we have exhausted the possibilities of analysis, the limits of Analytical science (Hegel 2001, par. 1720-1764). Hegel states "Synthetic cognition aims at the comprehension of what is, that is, at grasping the multiplicity of determinations in their unity. It is therefore the second premise of the syllogism in which the diverse as such is related". "Synthetic cognition" is presented by Hegel in contradistinction to "Analytic cognition". (Kant 1787) considered time an a-priori of knowledge, and it is certainly an a-priori for the adult as well as space is. Piaget taught us that the genesis of the notions of space and time is found in the early childhood (Piaget 1999). Accounting for this philosophical tradition, we call dialectical openings to knowledge the dialectical constructions that set up the basic elements for the knowledge of Nature, such as time, space and object. In such a way, we emphasize their synthetic origin.
} 
it should be understood in its intuitive form, later to be formalised in a compatible form. We can identify the dialectical pairs ego-alter and change-permanence (time-identity). As for space, considering that it implies the concept of distance and with it the idea of what is within reach and what belongs to our world but cannot be reached without effort, it is the result of the dialectic near me (reachable) and at a distance (not reachable, i.e., not near me). Notice that the notion of oppositions as first principles is a subject already considered in Aristotle's Physics (1994-2010), also referring to previous philosophers.

Notation 1 In this section we will try as much as possible to use superindices $a, b$ to indicate subjective (i.e., depending on arbitrary decisions) concepts, quantities, etc., while related objective entities will have no superindex.

\subsection{Space and Time}

\subsubsection{Space}

Space is spontaneously conceived by children along the construction of reality (Piaget 1999). It is not possible to speak separately of object, space, time and ego, because the construction produces all of them as a single dialectic system (we could try to straighten this matter up philosophically, although this would not be loyal to the child's structuring of thought). By exercising our memory we perceive some degree of permanence of ourselves, ego, and thus an idea of identity. The real world acquires continuity, we do not longer watch the movie frame by frame. Together with the recognition of ourselves comes alter, i.e., not-ego, and with the help of our memory some sort of permanency of alter emerges as well, the object that later, stripped of all other characteristics will be the "body" referenced in physics. Therefore, objects, people and other things acquire some sort of identity, inasmuch as we remove some attributes of them, mainly positions in space. Then, if an object (or ourselves) is in a place in one movie-frame and in a different place in another movie-frame, we no longer say they are different objects but rather that there was a change in the positions as well as some permanence: the object itself. The sequence of changes in places is the primary idea of time. Much later the child will conceive herself as being of the same condition than family, pets and toys, this is, she will place herself in the space. Space, with its implication of distance (a concept that is easy to root in sensorymotive intelligence) is opposed to the unity of the cosmos of the child.

The perceived space-time, centered in the observer (ego) always has ego distinguishing a reference point. This primary, subjective, notion of space is compatible with an empty space, a holder of objects, an idea that in physics goes back, at least, to Newton. Emptiness is in fact a resource for the subsequent suppression of the observer. By NAP, the required objectivity of the laws of physics manifests in that no law is objective if it depends on ego. The mathematisation of this idea is expressed by Eq. (2.1), where the group $T$ corresponds to spatial translations and rotations.

Axiom 1 A set of three orthogonal directions in the real (sensible) space is selected and represented by the symbols $\mathbf{e}_{i}$. Any objective position is then represented by $x_{A B}=\sum_{i} x_{i} \mathbf{e}_{i}$ with $x_{i}$ real numbers.

Objective space is hence built following Descartes and is represented as a vector space. Any other choice of reference vectors can be related to the initial one by a linear 
transformation, $\mathbf{e}_{j}^{\prime}=\sum_{i} R_{j i} \mathbf{e}_{i}$. It follows that $\sum_{j} x_{j}^{\prime} \mathbf{e}_{j}^{\prime}=\sum_{i} x_{i} \mathbf{e}_{i}$ for any objective position, hence $x_{j}^{\prime}=\sum_{i}\left[R^{-1}\right]_{i j} x_{i}$. The observer can then select a reference point and three independent orientations to describe the position of objects in the world, yet leaving the distance as objective. The class of equivalence thus generated is described by the group $I O(3)$, the group of isometries (translations, rotations and reflections).

Subjective space, being empty, provides no way of distinguishing one direction from another or one reference point from another. As a consequence of NAP, subjective space is then isotropic and homogeneous. We notice that objective space bears some relation to Leibniz' relational space, while subjective space is related to Newton's relative space (Ballard 1960).

We recall that Axioms correspond to intuitions based upon experience, the Cartesian space agrees with the experience of the child and of the scientist in Newton times. Furthermore, the analytical properties of e.g., Riemannian spaces are based upon differentiable manifolds which in turn rest upon collections of local charts of Cartesian type, a fact that speaks loudly about which one is the intuitive space.

\subsubsection{Time}

Unlike space, time is undoubtedly related to changes, sequences of changes, rapidity and causal relations (Piaget 1999). This is the genetic episteme of time; to call "time" any other kind of object is simply to ask for confusion ${ }^{5}$

\footnotetext{
${ }^{5}$ Piaget arrived to his view about the genesis of the concept of time after a series of experiments. The experiments are conveniently collected in (Piaget 1978). The notion of speed, at least in the form of faster and slower, precedes the notion of time: "From the point of view of immediate experience, the child succeeds very soon in estimating speeds of which he has direct awareness, the spaces traversed in an identical time or the "before" and "after" in arrival at a goal in cases of trajectories of the same length. But there is a considerable gap between this and a dissociation of the notion of speed to extract a measurement of time, for this would involve replacing the direct intuitions peculiar to the elementary accommodation of thought to things by a system of relations involving a constructive assimilation." (Piaget 1999, p. 383). Aristotle relates changes in position, velocity and time. In modern notation we could write (for finite $v>0$ )
}

$$
t-t_{A}=\int_{x_{A}}^{x} \frac{v \cdot d x}{|v|^{2}}=\int_{x_{A}}^{x} \frac{|d x|}{|v|} .
$$

We can move back to the perception of the child by making $v$ constant. Thus, change in position offers a clock, and since space is assumed to be continuous, so is time (an old reasoning already present in Aristotle). As long as the moon revolves around the Earth, we have change and we have time. No interval of time will ever be empty of changes. Other points of view regarding time have been put forward and continue to exist. For example, time has been conceived geometrically as well, taking such character in Special Relativity. Under the geometrical conception McTaggart has even argued that time is unreal (McTaggart 1908). The incompatibility of the perceived time with the time of Relativity resulting from Gödel work (1949) was discussed and asserted by Bell (2002). Here, a distinction has to be made. On one hand Piaget's view seeks the genesis of time (and the way we consider time in our daily facts) in the phenomena and its apprehension as idea. On the other hand, there are instrumental beliefs whose roots are nurtured by the mud of speculative material entities such as the $x$ ther and the electrical fluid veiled to us by mathematical axioms. The different ideas revolving around the notion of time might originate in overlooked assumptions of the idealisation method: when we proceed through successive idealisations, the order among them matters. Dynamical instabilities and irreversibility (entropy) are the result of first considering the limit of sufficiently long times and subsequently(if needed) the limit of infinite measurement precision (Arnold and Avez 1968). Deterministic, time-reversible dynamics rests on taking these limits in reverse order. The school of Brussels showed that, for some systems, irreversibility and time-reversal possibilities respond to different choices of representation (Goldstein et al. 1981) connected by transformation groups. The truth is that we have access to finite precision and finite time lapses, while the rest is only part of the processes of ideation. The arbitrariness that NAP tries to remove may take subtle and unexpected shapes. 
Time is the word we use to express our perception of change; it is change in its most abstract form. Aristotle indicates "without change there is no time" (1994-2010, Book IV, Ch. 11).

The perception of change we use to construct our intuition of time rests on natural processes. An invariant characteristic of our perception of Nature is that experienced processes have beginning and end. (Husserl 1983, Matter of fact) states "The founding cognitional act of experiencing posit something real individually; they posit it as something factually existing spatio-temporally, as something that is at this temporal locus, that has this duration of its own and a reality content which, with respect to its essence, could just as well have been at any other temporal locus."

Time is a verbalisation of the dialectical tension of the pair being/not-being. In principle, time is associated to objects (or measured) by the order of the sequence of changes (between sundown and sunrise the rooster sings, and if the rooster wakes me up every morning, it sings before-or rather while-I wake up, because this song is (part of) the cause of my awakening). Piaget (1999, Ch. 4) discusses the development of the notion of time in the child beginning from "As early as his reflex activity and the formation of his first habits, the nursling shows himself capable of two operations which concern the elaboration of the temporal series. In the first place, he knows how to coordinate his movements in time and to perform certain acts before others in regular order. For instance, he knows how to open his mouth and seek contact before sucking, how to steer his hand to his mouth and even his mouth to his thumb before putting the thumb between his lips, etc." The child proceeds (always according to Piaget) in five stages to develop his notion of time, always based on series. Starting at the third stage memory plays an increasingly relevant role.

One striking difference between time and space from the psychogenetic point of view is that while for the change of position in space of an object there is a possible operation that reverts the change, there is no operation capable to revert the temporal order of events. Unlike distance, that reaches us as a perception resulting from the telemetry of our binocular sight, time does not reach us as a perception, but it is rather the result of memory, a log of changes and a logical process that discriminates between the relative order of events. It follows that time is measured by comparing sequences of changes. By NAP, any transformation relating time-perceptions of different references (egos) is constrained to preserve this ordering. Therefore, while arbitrary individual subjective time may differ among observers, they are all related by strictly monotonic (bijective) mappings. The underlying group is the set of strictly increasing continuous functions $f: R \mapsto R$ with standard composition of functions as the group product.

Axiom 2 The order between the events (the various determinants and effects) involved in a causative relation is fixed.

This axiom relates to the concept of "determinism" discussed around Equation (A) in Russell (1912-1913, p. 199). In simpler words, first the vase falls, then it breaks. It is a vase as long as there exists a particular cohesion in the material. The alteration produced by the impact reorders the material in smaller pieces. Hence, without fall there is no impact, without impact no change in material stress. Causes here are gravitation and the change in material stress. Determinants are fall and impact, while breakup (rupture) is an effect.

When we measure the times of a phenomenon, say the change in position of a body, we use changes not involved in the process as references for time. Indeed, we resort to 
the idealised and imagined order of all events of the universe, encompassing all possible changes, leaving no "room" between them since a time without changes is a contradiction in the terms. ${ }^{6}$ In mathematical terms, time is well represented by a one dimensional mathematical space such as the real numbers, a notion that we have been using and will continue to use, along this work. Time-intervals are referred to this "background" of events that are present irrespective of the phenomenon in study. This background of changes constitutes a clock. We try to use as clocks those devices or observations that appear to us as regular. As long as we cannot present evidence that a process runs faster in one circumstance or another, we expect the relative order between changes in the clock and changes in the phenomena to remain the same, hence

Assumption 1 An objective time can be defined by convening on a process to define a time-unit.

Absolute time, a time encompassing all changes, appears to us in the same manner in which ego, alter, object and space emerge in the development of the child to produce a useful organisation of the world. The laws of physics and absolute time emerge in the construction of physics at the same step as a consequence of the same class of dialectic opening that creates the terms of an opposition that produces understanding. In this case, system-environment (not system) implies absolute time.

Definition 2 (Events) An event is a change in the sensible world that occurs in a relatively short interval of time and as such is idealised as instantaneous. We remove from the event the determinations of time and space, which are thought of as its circumstances. Thus, events occur at a given location and a given time.

\subsection{Observers}

The observer (ego) describes the world by measuring all distances with respect to a point of her/his election. We use the notation $x_{A}^{a}$ for the position of body $A$ as measured by observer $a$. When facing the need of relating positions of different observed bodies, conforming to NAP and the search for an objective description of physical entities, we define:

Definition 3 (relative position): We call $x_{A B}=x_{A}^{a}-x_{B}^{a}$ the relative position between observed bodies $A$ and $B$, as seen from the $e g o a$ at a given moment.

Corollary 1 It is a demand of objectivity (by NAP) that $x_{A}^{a}-x_{B}^{a}=x_{A}^{b}-x_{B}^{b}$ (hence, there is no superindex in $x_{A B}$ ).

\footnotetext{
${ }^{6}$ If time is conceived as holding a series of events, we may ask whether it is possible to have a time interval without changes or events (since each event represents a change). During a time without changes there would be no heart-beats of the observer, no movement of any object in the universe, no change that could be used as clock, etc. Between the two encompassing events of time without change there would be no way to measure time since measuring it requires something that changes. We would have then proposed that measurable (sensible) time differs from "true time" by an unaccountable amount, yet all observers will measure zero time identically since it is a property of monotonic functions to map open intervals into open intervals. Hence, a time interval without changes contradicts the early determination of time as associated to movement (change of position in space) and being therefore continuous.
} 
In a sense related to Leibniz, objective space is relational. Indeed, one may suspect that vector spaces have been constructed to this end. The group relating the arbitrary choices of different observers is $I O(3)$, the semi-direct product of isometries and translations.

In the sequel, we will call subjective the space created by an arbitrary choice of reference point and base vectors orientations.

Definition 4 Relative velocity is the rate of change of relative position between two bodies with respect to the change in time,

$$
v_{A B}=\frac{d\left(x_{A}^{a}-x_{B}^{a}\right)}{d t}=\lim _{\Delta t \rightarrow 0} \frac{\left(x_{A}^{a}(t+\Delta t)-x_{B}^{a}(t+\Delta t)\right)-\left(x_{A}^{a}(t)-x_{B}^{a}(t)\right)}{\Delta t} .
$$

\subsection{Subjective Velocity and Galileo Transformations}

The relative velocity between the reference point chosen by the observer $a$ and a body $A$, follows Definition 4,

$$
v_{A a}=\frac{d\left(x_{A}^{a}-x_{a}^{a}\right)}{d t}=\lim _{\Delta t \rightarrow 0} \frac{\left(x_{A}^{a}(t+\Delta t)-x_{a}^{a}(t+\Delta t)\right)-\left(x_{A}^{a}(t)-x_{a}^{a}(t)\right)}{\Delta t} .
$$

We now focus on the subjective operation consisting in setting $x_{a}^{a}(t+\Delta t)-x_{a}^{a}(t)=0^{a}$ and similarly $v_{a}^{a}=\lim _{\Delta t \rightarrow 0} \frac{x_{a}^{a}(t+\Delta t)-x_{a}^{a}(t)}{\Delta t}=0^{a}$, this is to say that for the observer, the point designated as reference by her/his arbitrary decision does not move (we have added the superscript $a$ to the zero to indicate the subjectivity). Now the relative velocity reads

$$
v_{A a}=\frac{d\left(x_{A}^{a}-x_{a}^{a}\right)}{d t}=\lim _{\Delta t \rightarrow 0} \frac{x_{A}^{a}(t+\Delta t)-x_{A}^{a}(t)}{\Delta t}-0^{a} \equiv v_{A}^{a}-0^{a},
$$

where we call $v_{A}^{a}$ the subjective velocity of $A$ as established by observer $a$.

Proposition 1 The Galilean transformation between observers (reference points) $a$ and $b$ is given by,

$$
v_{A}^{a}=v_{A}^{b}+v_{b a} .
$$

It is an operation that belongs to the group associated by NAP to the concept of subjective velocity.

Proof We begin by writing the equality

$$
x_{A}^{a}(t+d t)-x_{A}^{a}(t)-\left(x_{a}^{a}(t+d t)-x_{a}^{a}(t)\right)=x_{A}^{b}(t+d t)-x_{A}^{b}(t)-\left(x_{a}^{b}(t+d t)-x_{a}^{b}(t)\right),
$$

which after a rearrangement reads

$$
\left(x_{A}^{a}(t+d t)-x_{A}^{a}(t)\right)-0^{a}=\left(x_{A}^{b}(t+d t)-x_{A}^{b}(t)\right)-0^{b}+0^{b}-\left(x_{a}^{b}(t+d t)-x_{a}^{b}(t)\right) .
$$

Next we observe that

$0^{b}-\left(x_{a}^{b}(t+d t)-x_{a}^{b}(t)\right)=\left(x_{b}^{b}(t+d t)-x_{a}^{b}(t+d t)\right)-\left(x_{b}^{b}(t)-x_{a}^{b}(t)\right)=x_{b a}(t+d t)-x_{b a}(t)$,

with $x_{b a}$ an objective relative distance. We then have 


$$
\left(x_{A}^{a}(t+d t)-x_{A}^{a}(t)\right)-0^{a}=\left(x_{A}^{b}(t+d t)-x_{A}^{b}(t)\right)+\left(x_{a b}(t+d t)-x_{a b}(t)\right)-0^{b} .
$$

Dropping the zeroes, dividing by $d t$ and taking the limit $d t \rightarrow 0$, we obtain the Galilean transformation between observers. We further notice that the limit is not a necessary step.

Lemma 1 Galilean transformations form a group having vector addition as internal operation.

Proof Repeated use of Proposition 1 gives,

$$
\begin{aligned}
v_{A}^{a} & =v_{A}^{b}+v_{b a} \\
& =\left(v_{A}^{c}+v_{c b}\right)+v_{b a} \\
& =v_{A}^{c}+\left(v_{c b}+v_{b a}\right)=v_{A}^{c}+v_{c a} .
\end{aligned}
$$

\section{The Law of Inertia}

\subsection{Laws of Nature}

The meaning of laws of Nature deserves some examination. In western culture before the Enlightenment, it remitted us to God's blueprints for the universe as in the early times of Descartes, Newton and Leibniz. After the Enlightenment, the laws of Nature must rest on reason (Kant 1783, 1798). The question 'What is a law of Nature?' has been extensively debated in contemporary philosophy of science. Here we will not focus on what makes a statement to be a natural law. Nevertheless, no matter how that question is answered, certain general features of laws can be recognised. The laws of Nature correspond to fundamental relations in situations in which a small (minimal) portion of the universe, the system, is considered (through a process of idealisation) as isolated from its environment, i.e., the complement in the Universe of the ideally-isolated system. Such a notion implies that the internal organisation of the system — which the law will make explicit—, must be independent of the environment since this is the nature of the concept we are seeking. Hence, the law must hold with independence of the relative location of the system with respect to the environment and shall not be affected by the background of changes. In our perspective, there are no laws of Nature but rather laws for the understanding of Nature, which themselves are subject to the laws of reasoning and include in their ontogeny both experience and usefulness: the object and the subject.

\subsection{The Law of Inertia}

Definition 5 An isolated body is an idealisation consisting in extrapolating the (shorttime) motion of bodies that are perceived to be not interacting with other bodies.

This perception could originate in the fact that when the distance between bodies is sufficiently large or the time of the observation is sufficiently short, interactions do not show any appreciable effect (i.e., it is an idealisation). 
An isolated body can be regarded as being alone in the universe. As such, it defines by itself a privileged place and reference point. However, when we deal with several ideally isolated bodies, we must consider the problem of their changing (relative) distances (which is a result of the same idealising process). By NAP, the description given from the perspective of an isolated body must be equivalent to the description given by any other isolated body inasmuch as the particularity of the description is only that the observer is isolated (i.e., not influenced in its motion by other bodies). The condition of a body as isolated is a condition of permanence, hence, when compared with time (the measure of change) it has to be represented by a zero derivative of its state. Therefore, we define,

Definition 6 Inertial set is the collection of isolated bodies. The inertial class of observers consists of those observers that use an element of the inertial set as point of reference.

When seeking a fundamental law for isolated bodies we must consider first the possibility of giving them fixed relative positions, but such a law contradicts our current perceptions (we recall that in the Aristotelian physics the motion of bodies required causes, i.e., "forces").

Assumption 2 There exist at least two isolated bodies that are not at rest relative to each other.

The next possibility to be considered is:

\section{Lemma 2 Isolated bodies move with constant relative velocity.}

Proof Consider the law of motion of an isolated body, $A$, as described by an observer $a$ in the inertial class. By Assumption 2 the law of motion cannot be $\frac{d}{d t} x_{A a}=0$. The most general law of second order is

$$
\frac{d^{2}}{d t^{2}} x_{A a}=\alpha\left(x_{A a}, v_{A a}, t\right)
$$

However, the law must be the same for all times since there is no privileged time, $\alpha\left(x_{A a}, v_{A a}, t\right)=\alpha\left(x_{A a}, v_{A a}, 0\right)$. Additionally, by NAP, a second observer that has selected its reference position at a fixed distance from $a$ must produce the same law. It follows that the law cannot depend on $x_{A a}$ and by the same reasoning an observer that moves with constant relative speed with respect to $a$ must observe the same law, hence $\alpha\left(x_{A a}, v_{A a}, t\right)=\Upsilon$, being $\Upsilon$ a constant vector. However, such constant would indicate a particular direction in space unless $\Upsilon=0$. Hence, we arrive to the expression

$$
\frac{d^{2}}{d t^{2}} x_{A a}=0
$$

that satisfies the established requisite of permanency.

Corollary 2 An inertial observer (i.e., an element of the inertial class) is one in which only interactions are associated to changes in the velocities.

Proof The result is just Lemma 2 in negative form. 
Theorem 1 Galilean transformations belong to the group of transformations mapping the position determinations of one inertial observer to those of another one.

Proof By Corollary 2 and Lemma 2 two inertial observers behave as two isolated bodies with constant (objective) relative velocity. The subjective velocities determined by each system are related via Proposition 1.

It is important to notice that Lemma 2 has an equivalent statement in terms of subjective space and time. The proof is based on the homogeneous and isotropic character of the subjective space.

Notice that the inertial observer is a resource of the subjective point of view, since the objective presentation focuses only in relations. In terms of the objective presentation if two bodies present a relative velocity that changes with time, then both of them cannot belong in the inertial class, i.e., at least one of them must be in interaction with something else. It cannot be ruled out that both of them interact and none is in the inertial class. Hence we have,

Corollary 3 The relative acceleration $\alpha_{B A}$, of the body $B$ with respect to an isolated body, $A$, depends only on the relative positions and velocities of the interacting material bodies. $\alpha_{B A} \equiv \alpha_{B A}\left(x_{B C}, v_{B C}\right.$; interaction attributes $)$, where $C$ is some other body in interaction with $B$.

\section{Interacting Bodies}

We have shown that the only law of motion compatible with isolated bodies is the law of inertia. It is time to consider bodies with interactions. The same law in its negative form is: accelerations (i.e., changes in the instantaneous velocity) are the result of interactions between bodies. The simplest form of interaction to be considered involves just two material bodies. Consider an observer in the inertial class (hence, it does not interact with the bodies under consideration). From its perspective, if bodies $A$ and $B$ do not follow the inertial law, they must be interacting, hence the minimal setup for non-inertial motion requires two bodies that are described by the inertial (isolated) observer, which establishes the following relations:

$$
\begin{aligned}
\frac{d}{d t} v_{A}^{a} & =\alpha_{A}^{a} \\
\frac{d}{d t} v_{B}^{a} & =\alpha_{B}^{a} \\
\frac{d}{d t}\left(v_{A}^{a}-v_{B}^{a}\right) & =\frac{d}{d t} v_{A B}=\alpha_{A B},
\end{aligned}
$$

where $\alpha_{A B}$ is the (objective) relative acceleration of body $A$ with respect to body $B$.

Assumption 3 Accelerations are additive i.e., for any inertial observer $a$, the acceleration produced onto a material body (A) jointly by bodies (B) and (C), given that B and $\mathrm{C}$ do not influence each other, satisfies $\alpha_{A}^{a}(B, C)=\alpha_{A}^{a}(B)+\alpha_{A}^{a}(C)$, i.e., it is the sum of the accelerations produced by individual interactions of A with (B) and with (C). 
Following Newton,

Assumption 4 We consider in the sequel interactions of instantaneous action at a distance.

Other types of interactions may be imagined, but we are focusing on Newton's programme. This assumption was suggested by observation. For gravitation, it has so far resisted refutation. Indeed, several experimental measurements indicate that in classical terms the "speed of gravity" must be larger than $10^{8} C$ (being $C$ the speed of light), the first argument going back to Laplace (Van Flandern 1998).

\subsection{Gravitation and Mass}

The fundamental interaction between two bodies, the one that is always present and cannot be compensated, is called gravitation of matter. A fundamental ingredient at this point is Galileo's proof that all bodies experience the same acceleration in vertical motion (Galilei 1914, Third day, p. 173-). This achievement rests both on the logical need of investigating accelerated bodies after inquiring about non-accelerated bodies (Eq. 4.1) and on experimental observation.

Assumption 5 The gravitational acceleration $\alpha_{A}^{a}(B)$ produced onto a material body $(A)$ by a given one $(B)$ is proportional to a characteristic of the body $B$ named the mass (all other circumstances being identical). The mass of body $B$ is an objective property (i.e., the same, up to choice of units, for any inertial observer).

In mathematical terms, the accelerations are, letting $g_{A}^{a}(B)$ summarise the rest of the dependencies,

$$
\begin{aligned}
& \alpha_{A}^{a}(B)=m_{B} g_{A}^{a}(B) \\
& \alpha_{B}^{a}(A)=m_{A} g_{B}^{a}(A) .
\end{aligned}
$$

Corollary 4 The mass of an aggregation of matter is the aggregated mass of the parts.

Notation 2 From here on, we will drop the index of ego, since we are dealing with just one observer belonging to the inertial class. For example, we write $\alpha_{A} \equiv \alpha_{A}^{a}$. However, quantities with two "body"-subindices, such as $f_{A B}$ are always objective. ${ }^{7}$

Assumption 6 The gravitational interaction depends only on relative position.

With this we mean that letting $x_{A B}=x_{A}-x_{B}$, then $g_{A}(B)=x_{A B} \phi$, where $\phi$ is a scalar function that may depend on the length of the vector $x_{A B}$. When considering the

\footnotetext{
7 Binkoski (2016) argues that with three relational hypotheses it is possible to distinguish inertial systems from non inertial, while "Galilean relational space-time is too weak of a structure to support a relational interpretation of classical mechanics." At this point of our formulation it is clear that Newtonian mechanics considers an (external) observer, something what is necessarily non-relational. In more general terms, the dialectic objective-subjective (attaining objectivity by negating, going across, subjectivity) that we have presented solves the apparent opposition between Newton and Leibniz. This solution is reached by means of a critical reflection, not by adding ad-hoc postulates aiming to obtain some desired relation (what would amount to resort to instrumental reason).
} 
gravitational interaction of two bodies, $A$, localised in $x_{A}$ and, $B$, localised in $x_{B}$ according to an inertial observer, we notice that exchanging attributes between bodies is the same as exchanging positions, hence an immediate corollary of this assumption is that $g_{A}(B)=-g_{B}(A)$. As a consequence, to render this symmetry explicit rather than acceleration it is convenient to consider

Definition $7 F_{A B}=m_{A} \alpha_{A}(B)=m_{A} m_{B} g_{A}(B)$ is called gravitational force.

A related quantity is

Definition 8 The linear momentum of a material body is the product of its mass times its velocity $p=m v$.

Thus, the gravitational interaction is described by the symmetric form

$$
\begin{aligned}
& \frac{d}{d t} p_{A}=m_{A} \frac{d}{d t} v_{A}=m_{A} \alpha_{A}(B) \equiv F_{A B}=m_{A} m_{B} g_{A}(B) \\
& \frac{d}{d t} p_{B}=m_{B} \frac{d}{d t} v_{B}=m_{B} \alpha_{B}(A) \equiv F_{B A}=m_{B} m_{A} g_{B}(A),
\end{aligned}
$$

the latter being a definition of the concept of gravitational force and of force, the cause for the changes in momentum.

Corollary 5 The total momentum $p_{A}+p_{B}$ is constant in time when the bodies interact gravitationally, i.e.,

$$
\frac{d}{d t}\left(p_{A}+p_{B}\right)=\frac{d}{d t} p_{A}+\frac{d}{d t} p_{B}=m_{A} m_{B} g_{A}(B)+m_{B} m_{A} g_{B}(A)=0 .
$$

The previous corollary is an immediate consequence of Assumption 6, i.e., of $g_{A}(B)=-g_{B}(A)$. In Classical Mechanics it is called Newton's third law and also Principle of Action and Reaction.

\subsection{Other Interactions}

Let us now consider other interactions following the previous Newtonian scheme. Assumptions 5 and 6 are too narrow to encompass other types of interactions, despite its adequacy for gravitation. We rephrase them in a more general setting as

Assumption $5^{\prime}$ The defining property $Q_{i}$ is additive for $\mathrm{f}$, i.e.,

$$
f\left(Q_{1}+Q_{2}, x_{A}, \ldots, Q_{B}, x_{B}, \ldots\right)=f\left(Q_{1}, x_{A}, \ldots, Q_{B}, x_{B}, \ldots\right)+f\left(Q_{2}, x_{A}, \ldots, Q_{B}, x_{B}, \ldots\right) .
$$

Here $Q_{i}$ denotes the defining property of body $i$ associated to the interaction, i.e., the necessary information in order to determine the interaction, and $f$ is the associated force, i.e., a quantity representing the departure of bodies $A$ and $B$ from inertial motion. For example, $Q$ stands for electric charge and $f$ for the Coulomb electrostatic force (see the final paragraph of Sect. 5.4 for further discussion). 
Assumption 6' We consider in the sequel interactions that depend only on relative position and relative velocity.

Newtonian mechanics is based on the generalisation of Definition 7 to other types of interactions, namely

Assumption 7 Force $f$ is the cause of acceleration. The latter is proportional to the ratio between force and mass.

We have already realised that a consequence of Lemma 2 is that the presence of accelerations is an indication (or a symptom) of the existence of interactions. This assumption states that the totality of the intervening interactions is exhausted in the acceleration, they are its cause. We should notice that this assumption must be contrasted with experiments. ${ }^{8}$ Hence, we write,

$$
\begin{aligned}
& m_{A} \frac{d^{2}}{d t^{2}} x_{A}=f\left(Q_{A}, x_{A}, \ldots, Q_{B}, x_{B}, \ldots\right) \\
& m_{B} \frac{d^{2}}{d t^{2}} x_{B}=k\left(Q_{A}, x_{A}, \ldots, Q_{B}, x_{B}, \ldots\right) .
\end{aligned}
$$

In this presentation there is no need to introduce inertial mass as something different from gravitatory mass. Gravitation is taken to be a fundamental (unavoidable) interaction and its associated mass is what enters in Eqs. (5.2 and 5.3). This is a consequence of the symmetry of the presentation, by which whatever pertains the gravitational interaction is explicitly displayed.

\subsubsection{Consequences}

The above symmetry considerations may be propounded for Eqs. (5.2 and 5.3). In fact, $f\left(Q_{A}, x_{A}, \ldots, Q_{B}, x_{B}, \ldots\right)$ is the force that the body with property $Q_{B}$ and geometrical parameters $x_{B}, \ldots$ exerts on the body with property $Q_{A}$ (and $x_{A}, \ldots$ ). The dots indicate that forces may depend on other geometrical properties than just position (velocity, for example). The quantity $k\left(Q_{A}, x_{A}, \ldots, Q_{B}, x_{B}, \ldots\right)$ is the corresponding force that $A$ exerts on $B$. It follows by NAP that

$$
k\left(Q_{A}, x_{A}, \ldots, Q_{B}, x_{B}, \ldots\right)=f\left(Q_{B}, x_{B}, \ldots, Q_{A}, x_{A}, \ldots\right) .
$$

Lemma 3 Under the previous assumptions, for a pair of bodies $A$ and $B$ in interaction, the forces $f$ (force on A caused by B) and $k$ (force on B caused by A) satisfy the Generalised Principle of Action and Reaction, namely

$$
\begin{gathered}
f=x_{A B} \phi_{e}+v_{A B} \phi_{s}+\left(x_{A B} \times v_{A B}\right) \phi_{\perp} \\
k=-x_{A B} \phi_{e}-v_{A B} \phi_{s}+\left(x_{A B} \times v_{A B}\right) \phi_{\perp},
\end{gathered}
$$

\footnotetext{
${ }^{8}$ For example, Weber's formalisation of Faraday's induction law (Weber 1846) requires for the induction (electromotive) force to depend on the acceleration (as Faraday's law also does). This may be interpreted as having a mass that depends on the relative state of motion of the interacting charges.
} 
where $\phi_{e}, \phi_{s}, \phi_{\perp}$ are scalar functions of relative position, relative velocity and possibly other parameters, $x_{A B}=x_{A}-x_{B}$ and $v_{A B}=v_{A}-v_{B}$.

Proof By Assumption $6^{\prime}$ there is no other geometric dependency and we can replace the occurrences of $x_{A}, x_{B}$ by $x_{A B}$ and those of $v_{A}, v_{B}$ by $v_{A B}$. Let $T$ denote the operation of exchanging the geometrical properties associated to bodies $A$ and $B$ (or, what is the same, switching their defining properties $Q_{i}$ ). We have, by NAP,

$$
\begin{aligned}
T f\left(Q_{1}, Q_{2}, x_{A B}, v_{A B}, \ldots, t\right) & =f\left(Q_{2}, Q_{1}, x_{A B}, v_{A B}, \ldots, t\right) \\
& =k\left(Q_{1}, Q_{2},-x_{A B},-v_{A B}, \ldots, t\right) \\
T k\left(Q_{1}, Q_{2},-x_{A B},-v_{A B}, \ldots, t\right) & =k\left(Q_{2}, Q_{1},-x_{A B},-v_{A B}, \ldots, t\right) \\
& =f\left(Q_{1}, Q_{2}, x_{A B}, v_{A B}, \ldots, t\right)
\end{aligned}
$$

and consequently $T^{2}=I d$. The most general expression for a force between two interacting bodies depending as vector only on the vectors $x_{A B}$ and $v_{A B}$ is

$$
\begin{aligned}
& f\left(Q_{1}, Q_{2}, x_{A B}, v_{A B}, t\right)=x_{A B} \phi_{e}+v_{A B} \phi_{s}+\left(x_{A B} \times v_{A B}\right) \phi_{\perp} \\
& k\left(Q_{1}, Q_{2}, x_{A B}, v_{A B}, t\right)=x_{A B} \psi_{e}+v_{A B} \psi_{s}+\left(x_{A B} \times v_{A B}\right) \psi_{\perp},
\end{aligned}
$$

where the multiplicative factors $\phi_{e}, \phi_{s}, \phi_{\perp}, \psi_{e}, \psi_{s}, \psi_{\perp}$ are scalar functions of relative position, relative velocity and possibly other parameters. The reason is that relative position and relative velocity define only three vectors in space. In other words, $\phi_{e}$, etc., may depend on various properties (charge, velocity, etc.) but are scalar coefficients. The vector property of $f$ and $k$ is given by linear combinations of $x_{A B}, v_{A B}$ and $x_{A B} \times v_{A B}$. We recall that the vector $w \times z$ (called cross product or vector product of $w$ and $z$ ) has a direction that is perpendicular to both $w$ and $z$ and whenever $w$ and $z$ are collinear, $w \times z=0$.

Exchanging the properties $Q_{1}$ and $Q_{2}$ of $A$ and $B$ is equivalent to keeping the properties and exchanging their masses, positions and velocities. Again by NAP, from Eqs. (5.4 and 5.5) we have,

$$
\begin{aligned}
f\left(Q_{1}, Q_{2}, x_{A B}, v_{A B}, t\right) & =x_{A B} \phi_{e}+v_{A B} \phi_{s}+\left(x_{A B} \times v_{A B}\right) \phi_{\perp} \\
& =-x_{A B} \psi_{e}-v_{A B} \psi_{s}+\left(\left(-x_{A B}\right) \times\left(-v_{A B}\right)\right) \psi_{\perp} \\
& =-x_{A B} \psi_{e}-v_{A B} \psi_{s}+\left(x_{A B} \times v_{A B}\right) \psi_{\perp},
\end{aligned}
$$

which produces

$$
\phi_{e}=-\psi_{e}, \phi_{s}=-\psi_{s}, \phi_{\perp}=\psi_{\perp}
$$

Remark 1 Gravitation, as conceived by Newton, has vanishing $\phi_{s}$ and $\phi_{\perp}$.

Lemma 4 Under the Generalized Principle of Action and Reaction the total momentum, $p_{A}+p_{B}$, is constant in time if and only if $\phi_{\perp}=0$. 
Proof We just compute

$$
\begin{aligned}
\frac{d}{d t}\left(p_{A}+p_{B}\right)= & f+k \\
= & x_{A B}\left(\phi_{e}-\phi_{e}\right)+v_{A B}\left(\phi_{s}-\phi_{s}\right) \\
& +\left(x_{A B} \times v_{A B}\right)\left(\phi_{\perp}+\phi_{\perp}\right) \\
= & 2\left(x_{A B} \times v_{A B}\right) \phi_{\perp} .
\end{aligned}
$$

Moreover, if $(x-y)$ is parallel to $(u-v)$ (unidimensional relative motion) the actual value of $\phi_{\perp}$ is irrelevant and we can choose to set $\phi_{\perp}=0$ also in that situation.

We must notice that the total momentum as perceived by the observer is a conserved quantity if and only if $\phi_{\perp}=0$ for all forces in classical mechanics. The idea of conservation of total momentum is a consequence of Newtonian tradition and not a demand of reason. It must be established in every new theory of Nature as an additional assumption subject to empirical consideration.

Lemma 5 Under the Generalized Principle of Action and Reaction there is no internal torque if and only if $\phi_{S}=\phi_{\perp}=0$.

Proof Following Corollary 4, we compute, recalling that $\frac{d}{d t} v_{A B}=\alpha_{A B}=\frac{f}{m_{A}}-\frac{k}{m_{B}}$,

$$
\begin{aligned}
x_{A B} \times \frac{m_{A} m_{B}}{m_{A}+m_{B}} \frac{d}{d t} v_{A B}= & x_{A B} \times \frac{m_{A} m_{B}}{m_{A}+m_{B}}\left(\frac{f}{m_{A}}-\frac{k}{m_{B}}\right) \\
= & x_{A B} \times\left(x_{A B} \phi_{e}+v_{A B} \phi_{s}\right) \\
& +\left(\frac{m_{B}-m_{A}}{m_{A}+m_{B}}\right) x_{A B} \times\left(x_{A B} \times v_{A B}\right) \phi_{\perp} .
\end{aligned}
$$

The dependence on $\phi_{e}$ vanishes by the properties of the cross product. Since the remaining terms in the rhs have contributions in different directions,

$$
\frac{d}{d t}\left[x_{A B} \times \frac{m_{A} m_{B}}{m_{A}+m_{B}} v_{A B}\right]=0 \Leftrightarrow \phi_{s}=\phi_{\perp}=0 .
$$

In Classical Mechanics the quantity in square brackets is called angular momentum and its time-derivative is called torque.

\subsection{Central Forces}

An important issue in Classical Mechanics following the Newtonian tradition is that of central forces depending only on the relative distance. From the previous results, the following particular case can be highlighted: 
Lemma 6 Under the previous assumptions, if $\phi_{s}=0$ and $\phi_{e}=h\left(\left|x_{A B}\right|\right)$ (a scalar function of relative distance only) then there exists a dynamical quantity (called internal energy) that is constant in time.

Proof A standard computation using the relative (objective) quantities defined previously yields,

$$
\left(v_{A B}\right) \cdot \frac{d}{d t}\left(v_{A B}\right) \equiv \frac{1}{2} \frac{d}{d t}\left(v_{A B}\right)^{2}=\left(v_{A B}\right) \cdot\left(\frac{f}{m_{A}}-\frac{k}{m_{B}}\right),
$$

which by Eqs. (5.6 and 5.7) reads (since $v_{A B} \cdot\left(x_{A B} \times v_{A B}\right)=0$ ),

$$
\frac{1}{2} \frac{d}{d t}\left(v_{A B}\right)^{2}=\left(\frac{1}{m_{A}}+\frac{1}{m_{B}}\right) v_{A B} \cdot\left(x_{A B} \phi_{e}+v_{A B} \phi_{S}\right) .
$$

In other words, under the conditions of this Lemma, we have,

$$
\frac{1}{2} \frac{m_{A} m_{B}}{m_{A}+m_{B}} \frac{d}{d t}\left(v_{A B}\right)^{2}=h\left(\left|x_{A B}\right|\right) x_{A B} \cdot v_{A B} .
$$

We identify in the lhs the kinetic energy for the relative motion. Let $-V\left(\left|x_{A B}\right|\right)$ be such that $-\nabla V=h\left(\left|x_{A B}\right|\right) x_{A B}$. Then, $-\frac{d V}{d t}=h\left(\left|x_{A B}\right|\right) x_{A B} \cdot v_{A B}$ and therefore

$$
\frac{d}{d t}\left(\frac{1}{2} \frac{m_{A} m_{B}}{m_{A}+m_{B}}\left(v_{A B}\right)^{2}+V\left(\left|x_{A B}\right|\right)\right)=0 .
$$

The quantity $E=\frac{1}{2} \frac{m_{A} m_{B}}{m_{A}+m_{B}}\left(v_{A B}\right)^{2}+V\left(\left|x_{A B}\right|\right)$ is known in Classical Mechanics as internal energy.

\subsection{The Tension Between Inertial and Non-inertial}

One of the pillars of our understanding of Nature is observation, which invariably takes place on Earth (or since recently on its neighbouring galactic surroundings). Both the surface of the Earth and the Solar System are regarded as non-inertial references by Classical Mechanics. Indeed, we have no reasons to doubt of this non-inertiality since we detect its effects. However, since the Leibniz-Clarke discussion and lately Mach (see below) it has been an important issue the fact we have no means to decide whether a reference system is inertial (fully free from interactions) or not.

Despite this issue, physics has succeeded in conceiving different fundamental interactions, such as gravitation or the electrostatic interaction as described by Coulomb. One may wonder how this could be done in the first place, without having a clue about "how inertial" we are. The solution presented here finds its support on three concepts: (a) the process of idealisation described by Galileo, by which one identifies and eliminates from observation what is conceived to be foreign to the interaction under study, e.g., the effect of friction forces, or the presence of other interactions influencing both the system and the observer, (b) a fully-objective approach where the description of an interaction is performed using objective (invariant) quantities belonging to the interaction pair (relative positions, charges and the like) and (c) the honest effort to keep the description free from influences coming from 
the observer to the largest possible extent (this description is always provisional until a new, so-far neglected, influence of the observer is detected). We return to this issue in Sect. 6.1.

It is illustrative to consider the way in which the founders of electromagnetism studied the new interactions, an approach based on Assumption 3. Prior to experimenting with the new interaction, they let the system to be at rest in the observer's frame as a result of the equilibrium of the (other) present forces and accelerations. Next, the new influential condition was established and when a new equilibrium was reached they measured the balancing force judged to be equivalent (but opposite in sign) to the newly introduced force. Thus, forces are introduced as members of an equivalence class rather than by a direct application of the definition, i.e., no acceleration is truly measured in a first instance. Such were the methods of Coulomb and Ampère for example.

\section{On Newton's Laws of Classical Mechanics}

Those, like us, that have tried to reach a deeper understanding of Newton's ideas by reading the Principia, might have come to the conclusion that Newton left behind little or no clues on the fundamentals behind his axioms. In the Scholium to the Definitions (Newton 1687), Newton writes his famous notion of absolute space and time. Although he recognises them as related to the common intuition of "the vulgar" he proposes that the vulgar conceptions are imperfect images of absolute time and absolute space, a position that reminds us of the precedence of Platonic worlds, being the world of ideas the real world (Plato 2014). Needless to say, Newton relies on the vulgar notions to deliver and argument for his notions of space and time, place and motion. He indeed introduces without explicit recognition the idea of Galilean transformations: "Thus in a ship under sail, the relative place of a body is that part of the ship which the body possesses, [...] But real, absolute rest, is the continuance of the body in the same part of that immovable space in which the ship itself, its cavity, and all that it contains is moved. Wherefore if the earth is really at rest, the body which relatively rests in the ship, will really and absolutely move and with the same velocity which the ship has on the earth. But if the earth also moves the truly and absolute motion of the body will arise, partly from the true motion of the earth in immovable space; partly from the relative motion of the ship on the earth; and if the body moves also relatively in the ship; its true motion will arise, partly from the true motion of the earth in immovable space, and partly from the relative motions as well of the ship on the earth, as of the body on the ship;..." ((Motte) Newton 1687, p. 78) For Newton, it is relative space what can be conceived by the vulgar as fixed to the heavens: "All things are placed in time as to order of succession; and in space as to order of situation. It is from their essence or nature that they are places; and that the primary place of things should be movable, is absurd [...] But because the parts of space cannot be seen, or distinguished from one another by our senses, therefore in their stead we use sensible measures of them. For from the positions and distances of things from any body considered as immovable, we define all places; and then with respect to such places we estimate all motions...And so, instead of absolute places and motions we use relative ones, and that without any inconvenience in common affairs; but in philosophical disquisitions, we ought to abstract from our senses and consider things themselves, distinct from what are only sensible measures of them. For it may be that there is no body at rest to which the places and motions of others may be referred 
((Motte) Newton 1687, p. 79) (Cajori corrected the last phrase to: "And therefore, as it is possible, that in the remote region of fixed stars, and perhaps far beyond them, there may be some body absolutely at rest..." ((Mote-Cajori) Newton 1687)).

Newton's construction cannot come to an end unless the existence of an end, absolute space, is introduced ad-hoc. In terms of the present work, Newton's construction is moving from the subjectivity of one reference into the subjectivity of another, each one carrying its relative space. The sequence of subjective views can only finish in an objective view. Is God the observer of absolute space? Thus, rather than fixed to the heavens, absolute space is fixed to the Heavens, i.e., objective space would be God's perspective. The same sort of recursion is present in absolute time [see General Scholium, (Newton 1687, p. 503-)].

Concerning time, Newton did not conceive a method of measuring absolute time, but only approximations to the ideal time: "Absolute time, in astronomy, is distinguished from relative, by the equation or correction of the vulgar time. For the natural days are truly unequal, though they are commonly considered as equal, and used for a measure of time; astronomers correct this inequality for their more accurate deducing of the celestial motions. It may be, that there is no such thing as an equable motion, whereby time may be accurately measured. All motions may be accelerated and retarded; but the true, or equable, progress of absolute time is liable to no change." ((Motte) Newton 1687, p. 78)

Newton conceived absolute space and time as the limit of a process in which relative motions were added and clocks were perfected in their regularity. The opposite view of space in those years was that of Leibniz who sustained the relational view. We have shown in this work that both views are intimately related and that absolute space and time consist in a final attempt to maintain the observer in the scene. In our discussion, in contrast, subjective space and motion are the result of the introduction of the idealised observer in the scene. Newton's view of absolute time and space corresponds to the egocentric stage of the child, as observed by Piaget (1999, p. 367), later to be modified by the recognition of the other. It is important to notice that although relative space appears as objective, relative time appears only as intersubjective, being impossible for us to define an objective measure of time without an ad-hoc assumption, which in all cases amounts to construct a physical equivalent of our memory such as logging the tics of a clock or the oscillations of a pendulum.

In Principia Mathematica Newton founded the concept of mass on the intuition of weight: "The quantity of matter is the measure of the same, arising from its density and bulk conjointly [...] It is this quantity that I mean hereafter everywhere under the name of body or mass. And the same is known by the weight of each body; for it is proportional to the weight, as I have found by experiments on pendulums, very accurately made, which shall be shown hereafter." ((Motte) Newton 1687) Such an idea was later challenged in an a-posteriori empiricist view by (Mach 1919). We have shown in this work that Newton's perspective is proper to the construction of knowledge, this is, the genetic meaning of mass emerges from interactions. We realise that the gravitational interaction is the most notorious one, and pre-exists the concept of force. In the terms of Popper, Newton's theory is simpler than Mach's proposal, since it lends itself more generously to refutation (Popper 1959).

It is worth noting that Principia Mathematica dedicates special attention to the issue of relative vs. absolute rotations. A fundamental difference between rotational and translational motion is that rotations cannot be conceived without acceleration. In the Scholium to the first chapter Newton discusses centripetal forces: "A centripetal force is that by which bodies are drawn or impelled, or in any way tend, towards a point as to a centre. Of this sort is gravity" ((Motte) Newton 1687, p. 74) and he continues later "The effects which 
distinguish absolute from relative motion are, the forces of receding from the axis of circular motion. For there are no such forces in a circular motion purely relative, but in a true and absolute circular motion, they are greater or less, according to the quantity of the motion" ((Motte) Newton 1687, pp. 80-81). This view contrasts with Mach's attempts to wipe out the difference between relative and absolute rotations: "The principles of mechanics can, indeed, be so conceived, that even for relative rotations centrifugal forces arise" (Mach 1919, II.VI.5, p. 232). In that paragraph Mach remains within the kinematic aspect of relative rotations; what we may call the visual intuition of rotation. However, in the construction of knowledge, the child gradually adds other sensations to the visual, noticeably the physical effort required to sustain a rotation. The distinction relates to our experiences as children, with relative motion related to spinning around our vertical axis (while standing up) and absolute circular motion such as the one used to throw stones with a sling. While the visual appearance is equivalent, the complete sensorial experience is quite different. The sensorial difference has to be accounted for and Newton introduces the distinction between absolute and relative rotation. Mach makes an enormous metaphysical effort - thus betraying his own ideas - to disregard the difference in sensory effects arising in actual rotations as opposed to apparent ones (called in this context "relative" both by Newton and Mach). Poincaré (1913, Ch. VII) criticised Mach's view accurately indicating that in order to confuse apparent rotations with true ones, we need to assign to the apparent rotation a force which is contrary to intuition (it increases with distance), a sort of conspiracy of the rest of the universe to deceive the observer. Mach's attempt to suppress the subject comes short of Newton's insight about the difference between subjective and objective descriptions.

Poincaré (1913) insisted on this matter proposing for discussion an "Earth" always covered (clouded), so that celestial references could not be observed. The scientists of this "Earth" would discuss relative and absolute rotations. "In the theory of relative motion we observe, besides real forces, two imaginary forces, which we call ordinary centrifugal force and compounded centrifugal force. Our imaginary scientists can thus explain everything by looking upon these two forces as real, and they would not see in this a contradiction of the generalised principle of inertia, for these forces would depend, the one on the relative positions of the different parts of the system, such as real attractions, and the other on their relative velocities, as in the case of real frictions. Many difficulties, however, would before long awaken their attention. If they succeeded in realising an isolated system, the centre of gravity of this system would not have an approximately rectilinear path. They could invoke, to explain this fact, the centrifugal forces which they would regard as real, and which, no doubt, they would attribute to the mutual actions of the bodies-only they would not see these forces vanish at great distances - that is to say, in proportion as the isolation is better realised. Far from it. Centrifugal force increases indefinitely with distance. Already this difficulty would seem to them sufficiently serious, but it would not detain them for long. They would soon imagine some very subtle medium analogous to our ether, in which all bodies would be bathed, and which would exercise on them a repulsive action. But that is not all. Space is symmetrical-yet the laws of motion would present no symmetry. They should be able to distinguish between right and left. They would see, for instance, that cyclones always turn in the same direction, while for reasons of symmetry they should turn indifferently in any direction. If our scientists were able by dint of much hard work to make their universe perfectly symmetrical, this symmetry would not subsist, although there is no apparent reason why it should be disturbed in one direction more than in another. They would extract this from the situation no doubt they would invent something which would 
not be more extraordinary than the glass spheres of Ptolemy..." The discussion continues for long, mathematising this "Earth" until reaching a contradiction.

In terms of genetic epistemology a central place is taken by cognitive surpass ${ }^{9}$ shortly said, it consists in a reordering of previous knowledge, extending it into more general (universal) forms. The old knowledge, however, is not suppressed. It becomes rather a particular form of the new one, a form that supports as well the new structure of knowledge. Thus, the distinction about rotating around our axis and that of revolving a stone with a sling must be preserved. As indicated by Poincaré, Mach's posit destroys this difference. Still, the difference does not imply absolute space, because the latter means to conceive rotations with respect to something else, while for an extended body like the Earth rotations correspond to the movement of one part of the Earth around another. Hence, it is possible and simple to forget about the stars still preserving the eidetic seeing that originated the distinction. In the relationalist point of view, it can be said as well that the stone revolves around us, or both revolve around the common centre of mass, but in all cases, sensible forces are exerted between the revolving bodies, the bodies interact. The same can be said with respect to (parts of) the Earth, but the heavens have nothing to do with it.

\subsection{The Present View}

In this essay we explore the consequences of recognising our humanity, the acceptance of the undeniable fact that our thoughts have their grounds in the constructions we produced as babies and infants, later integrated with our cultural background. This structure is built always under the supervision of reason, since reason protects the unity of our conception of the world: the construction by dialectical oppositions that we have come to call understanding. Thus, the starting point of our construction is a principle of knowledge, an internal requirement of both what we are ready to accept as knowledge of the world and what has to be rejected as such. We named this principle the No Arbitrariness Principle (NAP). On the positive side, it enables us to pursue the quest of developing an objective knowledge of the world. Since knowledge means to put the input of the sensory system in correspondence with the organisational labour of our brain, ${ }^{10}$ truly objective knowledge can only be sought by a second motion in which the subject (the observer) removes her/himself from

\footnotetext{
9 Original in Spanish: rebasamiento cognoscitivo (Piaget and García 1989).

10 This point of view has several precedents. Kant (1787, Introduction) opens the book by saying "That all our knowledge begins with experience there can be no doubt. For how is it possible that the faculty of cognition should be awakened into exercise otherwise than by means of objects which affect our senses, and partly of themselves produce representations, partly rouse our powers of understanding into activity, to compare to connect, or to separate these, and so to convert the raw material of our sensuous impressions into a knowledge of objects, which is called experience?" and later "Understanding cannot intuite, and the sensuous faculty cannot think. In no other way than from the united operation of both, can knowledge arise". Husserl formulates similar ideas as "Isolated experience, even when it is accumulated, is still worth little to it. It is in the methodical disposition and connection of experiences, in the interplay of experience and thought, which has its logically rigid laws, that valid experience is distinguished from invalid, that each experience is accorded its level of validity, and that objectively valid knowledge as such, knowledge of nature, is worked out" (Husserl and Lauer 1956, p. 234). Piaget and Garcia state "Not only is there an absence of a clear frontier between the contributions of the subject and those of the object (since we can only know about interactions between the two) but in addition, it is only to the extent that logical and mathematical structures are applied that one can come to attain the object, and objectivity improves as a function of richer logico-mathematical structures. In fact, the elementary perceptual object is already partially 'logicized' from the start, even though much less 'objective' than the more elaborated object." (Piaget and García 1989, Introduction).
} 
the scene. This is the master plan of this work, attaining as much objectivity as possible by going across (transcending) subjectivity.

If space and time have their foundation in the early experiences of life, interactions are a rather different matter. Interactions are not objects in space. Forces are metaphysical entities responsible for the departure from inertial motion, they have no time since causes are only made apparent by their effects: the gravitational interaction is present before, during and after the fall of an object. Forces can only be recognised by their effects and characterised using the established framework in a process of inference. The first law of motion has then a stronger support that the other two; the result is independent of the way we characterise interactions. The second law and even more the third are in a good degree the result of promissory assumptions. Newton, in search of credibility for the principle of action and reaction, relies on contact forces that impress us in a sensory form ("If you press a stone with your finger, the finger is also pressed by the stone" ((Motte) Newton 1687)). In our discussion we explore to what extent the principle of action and reaction is a result of NAP, restricting our discussion to forces of instantaneous action. The result is a generalised principle of action and reaction. Thus, Newton's third law has a weaker logical support than the first and second laws. If classical forces are ever found that break reflection symmetry, we expect total linear momentum not to be conserved by the interaction.

Apart from assumptions of technical character and others suggested by observation, the present approach rests on a principle of knowledge (derived from NAP), namely that the relative properties of a pair of interacting objects depend only on the objects themselves and their interaction. Thus, we develop the concept of objective relative distance. Indeed, the construction rests on the resolution of the tension between objective and subjective. In this sense, we get past the traditional issue present in Leibniz, Newton and later Mach and Poincare (see below) about "absolute" and "relative" distances. The quest here is to objectivise relative distances. The present work disposes of absolute space, while retaining objectivity. Another basic underlying idea is that the concepts of space and time are different. Space is related to permanence and time to change. In particular, space and time are not interchangeable. This is based in the way we construct our knowledge.

\section{On the Laws of Nature}

In a society that rests on religious concepts it makes sense to consider that Nature is a creation of God and that She/He endowed Nature with laws. As religious beliefs decline and faith in our own strength grows, we come to accept that the laws of Nature are actually laws produced by humanity to understand Nature, they are laws for the understanding of Nature, constructs generated to organise the sensory world, the continuation at the level of civilisation of the work autonomously initiated by every child. As such, the laws of Nature are subject to a higher set of rules, those discovered by every member of our species in our early quest for survival. They include the conviction that "there is something out there" that reaches us through our senses (call it Nature) and therefore, the possibility of objective knowledge.

Unlike social rules, on which we agree in order to preserve energies for other relevant social matters and are established by consensus or power, unlike the "agreement on disagreeing" that makes room for cooperation by setting aside disputes, Truth is the only form of agreement with Nature, as Nature is not a social actor that can change behaviour or negotiate the rules. But Truth requires an agreement with our humanity as well. Thus, the 
laws of Nature or truth about Nature come as a result of the dialectic interplay between a universal (humanwise- or civilisationwise) subject and the universe of sensory observations, Nature. None of them can be absent in the laws of Nature. In turn, the universal subject requires a correspondence between the individuals, it requires intersubjectivity. Intersubjectivity sets the lower level of requisites on the side of the subject for calling something a law of Nature.

Much of our understanding of Nature comes from what we call "dialectical openings to knowledge". Thus, the recognition of ourselves requires the simultaneous recognition of not-ourselves, our environment. Likewise, the motion of isolated bodies, the inertial motion, has its necessary opponent in the motion of bodies that interact. At first sight, we could have introduced just unilateral action since the opponent of isolated is not-isolated, i.e., influenced by (or influencing) others. But such an idea contradicts the higher level of reasoning, the rules on rules, one of them being the No Arbitrariness Principle (NAP). Do we have a reason to support that one body can influence another without being influenced, that there is an asymmetry among bodies? So far, the answer is no.

The present approach is not just discursive, it is constructive as well. Let us see how it works on a much debated matter.

\subsection{The Speed of Light}

During a large part of the nineteenth century and well into the following century, a number of attempts to measure the speed of light and to understand its constancy were done. These attempts were mostly independent of the electromagnetic theory of light (at most, the wave-like properties of light were used). Experiments such as those by Fizeau in 1848, later developed and improved by Cornu in 1872-1874 (1876) (using a light source/detector, a rotating cogwheel and a mirror at rest relative to the source/detector) consider that there exists a light path and that the event "detect the light" occurs at a later time than the event "turn on the light". The measuring issue can hence be regarded as purely kinematical, although the connection between light and electrodynamics was advanced already in the mid 1800's. The final outcome of these efforts has been that the "speed of light" is constant and also that this constancy is incompatible with Newtonian mechanics. In this section we will show that under the present approach based on NAP the last assertion is incorrect.

\subsubsection{The View of this Work}

In the first place, we must raise the objection that light, being a perception resulting from the electromagnetic interaction, is not an object. Hence, what is meant by "the speed of light" needs to be explained. What is truly measured in Fizeau's or Cornu's experiments is a distance and a time-interval. The quotient of both has the dimensions of a velocity so we may agree to consider it a velocity. A view compatible with NAP, is to consider that all three events related to the measurements (emission, reflection and detection) are actually the result of a common cause, namely, the electromagnetic interaction.

Whatever we make in our interpretation of light, it is undeniable that what we have called the speed of light is the quotient between an objective distance and an intersubjective time interval. The result is objective (or rather intersubjective) and it is subject to the laws of transformations of objective quantities, this is, it is the same for all observers. 


\subsubsection{The (Traditional) Mediator View}

The main step in the traditional conception of light is to interpret the measured quotient in terms of the velocity of a body or a material wave, something that has a place in space. Since interactions are not matter, in principle this interpretation introduces a fundamental belief: interactions are mediated by substantial entities that as such have a place in space. As far as we know, neither this assumption nor its rational/philosophical basis is ever stated. Interactions do not have a place, interactions require two places and relative distances, such as the relative distance between source/detector and mirror. If we want to associate the events emission, reflection and detection in terms of traditional causal relations it will have to be in the order: turn on the light, reflect the light at the mirror, detect the light. The cause (in the modern sense discussed at the Introduction) of our detection of light cannot have ceased by the time of the detection. Since without turning on the light there is no light to be detected, and the same can be said when the mirror is not in place, both the presence of the mirror and the turning on of the light are within the determinants of the detected light. However, the observed events are not the causes in themselves since the first event might very well have ceased when the third event happens. Similarly, the detected light immediately associated with the turn-on event could only be a cause if it travelled (matter-like) in space. The interpretation in terms of a material analogous allows for questions that do not correspond to what it is truly measured.

Equating a subjective quantity such as $\left|v_{L}^{a}\right|$ (the velocity of an interpreted material point $L$ with respect to a reference $a$ ) with an objective quantity such as $C$ (namely a constant, and hence objective and invariant) is, to say the least, confusing. A possible construction could be to consider $\Delta t$ as subjective, since after all, absolute time was an assumption of Newtonian mechanics. If space is still to be conceived as absolute, then time might very well depend on the velocity of the reference system with respect to absolute space.

Let us work out an exercise along this line of thought. We will tentatively assume as a new axiom a deeply rooted belief that we are not going to offer for examination:

Tacit belief 1 Interactions are mediated by substantial entities that as such have a place in space and a velocity.

Then, if the substantial entity moves in absolute space with a characteristic velocity, we would expect that it appears to us with different (and measurable) relative velocities depending on the relative motion of our system of references with respect to absolute space. However, experiments (interpreted in this frame of mind) indicate otherwise: we measure always the same velocity. Therefore, absolute time, absolute space, these measurements and the present tacit belief have come into collision. We can ask ourselves what can we save from the wreckage? For the sake of the exercise, we insist in keeping the tacit belief alive. It has been argued that such a state of things is incompatible with Newtonian mechanics, but again, this is not the case. Is it possible that velocities transform between different reference systems satisfying the existence of a universal velocity and the structure of Newtonian mechanics? The following Theorems give an affirmative solution.

Definition 9 Let $U \in\left\{z \in \mathbb{R}^{3}:|z|<C\right\}$, with $|U|$ its Euclidean norm. Further, let $g(U)=G(|U|)$, where $G$ is a strictly increasing continuous function such that $G(0)=1$ and 
$\lim _{a \rightarrow C^{-}} G(a)=\infty$. We advance that $U$ will play the role of a velocity in the sequel. Then, for $|U|,|V|<C$ we define velocity addition $U \oplus V$ as

$$
U \oplus V=W \Leftrightarrow g(W) W=g(U) U+g(V) V,
$$

where addition in the rhs denotes standard vector addition in 3-space.

Theorem 2 (Nonlinear presentation of Galileo's group) Velocity addition has the following properties:

(a) $|U|,|V|<C \Rightarrow|W|<C$.

(b) $U \oplus 0=U$, i.e., 0 is the neutral element of velocity addition.

(c) $U \oplus V=V \oplus U$.

(d) For all $U$, the inverse of $U$ is $-U$, i.e., $U \oplus(-U)=0$.

(e) Associativity: $(W \oplus V) \oplus U=W \oplus(V \oplus U)$.

Proof For most statements it is a matter of doing the algebra. For statement a), the function $g(V)|V|$ is a bijection of the interval $[0, C)$ onto the nonnegative reals. $|U|,|V|<C \Rightarrow g(U) U+g(V) V<\infty$, hence there is a unique $|W|$ in $[0, C)$ such that $g(W) W=g(U) U+g(V) V$. Statements b), c) and d) follow from the definition. Regarding statement e), let $A=W \oplus V$ and $B=V \oplus U$. Applying the definition two times we have: $A \oplus U=g(A) A+g(U) U=g(W) W+g(V) V+g(U) U$. Similarly, $W \oplus B=g(W) W+g(B) B=g(W) W+g(V) V+g(U) U$.

In this exercise we consider relative position and velocity as primary concepts. The addition of time intervals has to be deduced from the concepts of relative position and velocity.

Definition 10 We call velocity $V$ (with $|V|<C$ ) the vector satisfying $\Delta x=V \Delta t$. For any reference system, we call $t$ subjective time, while $T=\frac{t}{g(V)}$ is called proper time.

The following Theorem indicates that while this way of thinking rests on a subjective time, there exists an underlying intersubjective time as long as space is objective.

Theorem 3 Let $O$ move with velocity $V_{1}$ with respect to $S$ and velocity $V_{2}$ relative to the observer $S^{\prime}$, while $S^{\prime}$ moves with velocity $V_{3}$ relative to $S$, such that $V_{1}=V_{2} \oplus V_{3}$. Let

$$
\begin{aligned}
\Delta x & =V_{1} \Delta t_{1} \\
\Delta x^{\prime} & =V_{2} \Delta t_{2} \\
\Delta_{S S^{\prime}} & =V_{3} \Delta t_{3}=-\Delta_{S^{\prime} S}
\end{aligned}
$$

and $\Delta T_{i}=\frac{\Delta t_{i}}{g\left(V_{i}\right)}$ for $i=1,2,3$. Then the proper time is invariant, $\Delta T_{1}=\Delta T_{2}=\Delta T_{3}$, if and only if distances in space are invariant.

Proof The distance between $S$ and $O$ can be determined both by $S$ and by $S^{\prime}$. Invariance of distances is expressed as

$$
d_{S O}=\Delta x=\Delta x^{\prime}+\Delta_{S S^{\prime}}=d_{S O}^{\prime}
$$


or, equivalently,

$$
V_{1}=V_{2} \frac{\Delta t_{2}}{\Delta t_{1}}+V_{3} \frac{\Delta t_{3}}{\Delta t_{1}}=V_{2} \frac{g\left(V_{2}\right)}{g\left(V_{1}\right)}+V_{3} \frac{g\left(V_{3}\right)}{g\left(V_{1}\right)} .
$$

The last equality follows from the law of addition of velocities. Invariance of distance holds for all velocities $U, V, W$ satisfying $V=U \oplus W$, if and only if

$$
\begin{aligned}
& \frac{\Delta t_{2}}{g\left(V_{2}\right)}=\frac{\Delta t_{1}}{g\left(V_{1}\right)} \\
& \frac{\Delta t_{3}}{g\left(V_{3}\right)}=\frac{\Delta t_{1}}{g\left(V_{1}\right)} .
\end{aligned}
$$

It follows that $\Delta T_{1}=\Delta T_{2}=\Delta T_{3}$. The reverse implication follows by assuming $\Delta T_{1}=\Delta T_{2}=\Delta T_{3}$ and applying the previous reasoning in reverse order to obtain $d_{S O}=d_{S O}^{\prime}$.

$\Delta T$ can be interpreted as the time measured by an observer moving with the object, since in such a case we have $g(0)=1$ and $\Delta T=\Delta t$.

As far as we know, the physics emerging from this picture has not been studied. Certainly, we may imagine other alternatives to exit the contradictory situation. For instance, we could drop entirely Tacit belief 1, as in the view of this work, or try other forms of reconciliation such as e.g., keeping Tacit belief 1 while dropping both absolute space and time, as well as relative distance. This part of the story is well known.

We have shown that the argued need to abandon the Newtonian frame upon the requirement of a constant speed of light is not mandatory. On the contrary, this course of action is related to a bolder decision: to associate interactions with the exchange of substantial entities (i.e., to consider light an object) and subsequently opting for a solution without all three of absolute space, absolute time and invariant relative distance.

\subsection{Poincaré's Principle of Relativity and Related Principles}

As we have already mentioned, the No Arbitrariness Principle relates to Leibniz' principle of sufficient reason. Mach also used the principle of sufficient reason in the form of a "principle of symmetry" (Mach 1919, p. 9). At the beginning of the twentieth century, a related principle became notorious, the principle of relativity presented by Poincare (1913, p. 107). "The motion of any system must obey the same laws, whether it be referred to fixed axes or to moving axes carrying along a rectilinear uniform motion. This is the principle of relative motion that forces upon us for two reasons: first, the commonest experiences confirm it, and second, the contrary hypothesis is singularly repugnant to the mind". Poincare hints us about the principle being a principle of knowledge ("not solely the result of experiment"), but does not elaborate further. As we have shown, Poincaré's principle belongs to the context of interactions, for in the description of interactions it should not matter which isolated body we take (arbitrarily) as reference for our calculations. This issue was advanced before in this work. Poincaré and Mach inherit the discussion between absolute and relative spaces already present in Leibniz-Clarke and Newton. They attempt to resolve this issue in different ways, most remarkably Mach reducing the cosmos to a set of empirical relations without subjects (observers). The present approach goes past this controversy by introducing the arbitrariness of the observer (and with her/him the concept of space) 
and subsequently eliminating the arbitrariness by focusing the understanding on objective or intersubjective elements. In short, this work takes sides for an objective and relative description of interactions, with the stress resting on its objective nature.

\section{Summary and Conclusions}

We have reconstructed Newtonian mechanics starting with a minimal realism (Lombardi and Pérez Ransanz 2012) that accepts as starting point the existence of a real, or objective, world. The analysis of this physical world, we called it Nature, proceeds through several constructive steps. Some of them consist in dialectical openings to understanding. The first opening is the opposition between objective and subjective: objective opposes not-objective, i.e., subjective, which is what comes from the knowing subject. Understanding Nature is the result of a dialectic interplay — or dialogue — between the knowing subject and the object being known. It is worth mentioning that the subject is not an individual in principle but rather humanity or society. Knowledge in this primary form cannot be objective, the subject needs to transcend (negate) her/his subjectivity if she/he is going to reach any possible objectivity. Hence, the subject establishes principles of reason such as the principle of sufficient reason, which we have recast in mathematical terms under the name of No Arbitrariness Principle. Actually, it is not the subject by her/himself who establishes the principle but the subject in relation to Nature. There is no form in which the dialectic objective-subjective can be separated giving pre-eminence to one of the terms.

NAP recognises the role of symmetry groups as a basic tool for understanding. Symmetries contribute with more than just aesthetic appealing (Wigner 1964); they express the permanence of the laws of Nature beyond the arbitrariness of the knowing subject. Moreover, NAP — or in a broader sense the principle of sufficient reason—might have represented an adaptive advantage for the early humanity, allowing to elaborate consequences of our relation with Nature.

NAP appears, at first sight, related to Kant's first formulation of the categorical imperative "Act only according to that maxim whereby you can, at the same time, will that it should become a universal law" (Kant 1993, 4:421). The requisite of universal application is precisely the action of removing all arbitrariness that may arise in the application of the maxim to different rational agents. A moral action must be free of arbitrariness. The associated set of arbitrariness usually considered is that of the agents subjected to the law. In more common terms, we can say: no rational justice exists if the laws do not pass the test of being equal for all citizens. We believe that NAP is a fundamental principle of rationality that emerges in different forms for different contexts.

Important contributions to mechanics come from the early childhood where the notions of time and space are formed as a consequence of the active adaptation of the child to her/his environment. In this stage of our construction, concepts point toward perceptions rather than to other concepts. With the opening produced by the concepts of isolated and interacting comes the first mechanical law, the only law acceptable to NAP for the condition of permanence of isolated objects. Repeated use of NAP in different contexts will finally unravel Newton's laws. But these laws require an increasing number of assumptions regarding Nature. Indeed, Newton's mechanics arises as the interplay of reason, idealisation and experience in our relation to Nature.

We address the laws of Nature not as rules proper to the objects, as in metaphysical realism (Lombardi and Pérez Ransanz 2012), but rather as laws for the understanding of 
Nature generated by the interplay between subject and object. This position becomes quite evident when Galilean transformations of velocities are not alleged to be experimental laws but rather considered as a requisite for the suppression of contributions arbitrarily introduced by the observer.

The adopted approach allows us to attain a higher level of consciousness on the strength of the different components that contribute to the construction of mechanics. There is a precedence between these components which can be better appreciated by considering the consequences of removing them: (a) the consequence of renouncing to logic and to the laws of understanding is not being able to understand the world, (b) renouncing to the early elaborations of primary concepts such as time and space leads to a dissociation between everyday life and physics, the latter becoming entirely pragmatic and justified a-posteriori (because it is convenient), (c) in contrast, modifying our temporary beliefs has no real cost other than the effort of reconstructing our understanding on a more solid basis. Moreover, the present approach allows for a critical view of further developments, since it opens for new alternatives to the current views in different issues, alternatives that we believe should be explored to decide their worth.

An important reflection corresponds to the use of tacit beliefs (also known, in mathematics, as hidden assumptions or hidden lemmas). Inasmuch as they are hidden and as such can escape the scrutiny of reason, they must be considered dangerous (Lissack 2017). They elude the principle of necessary reason and usually protect something that is not explicitly mentioned. In the case of Tacit belief 1, it hides the fact that we support the argument in a material model, be it wave or particle, on the basis that we cannot imagine it otherwise. Our imagination works with memories of the sensible world and in doing so it limits its value to the material experience. "Because we are not able to imagine otherwise" appears to us as an extremely weak argument: the confession of a limitation does not constitute a reason. We can explore the possibilities of the hidden assumption but we should be ready to abandon it if it comes into conflict with higher principles.

Scientific disciplines are not autonomous, they are ruled by reason, which is the only autonomous entity. The application of the principles of reason to understanding is known as critic. Critic in science needs not only be declared but it should be truly exercised. This work is indeed an exercise of critic. It seems proper to quote (Kant 1787, The discipline of reason) on this respect "...where reason is not held in a plain track by the influence of empirical or of pure intuition, that is, when it is employed in the transcendental sphere of pure conceptions, it stands in great need of discipline, to restrain its propensity to overstep the limits of possible experience and to keep it from wandering into error. In fact, the utility of the philosophy of pure reason is entirely of this negative character." The terms pure intuition and pure conceptions in Kant may be understood from: "Pure intuition consequently contains merely the form under which something is intuited, and pure conception only the form of the thought of an object. Only pure intuitions and pure conceptions are possible a priori; the empirical only a posteriori." (Kant 1787, p.75)

In conclusion, the construction of knowledge is not only a philosophical perspective but it is a constructive and critic method as well. Indeed, the history of knowledge has to recognise the contributions from metaphysical (unverifiable/irrefutable) ideas such as absolute space and later the reification of interactions. Changing the underlying metaphysics, great revolutions operate in physics, which is not automatically equal to great progresses. In these lines, recognising the need for introducing some metaphysics, the idea of a pluralist realism (Chang 2012; Lombardi and Pérez Ransanz 2012) should be considered not only as a description but rather as a programme. We might ask if the efforts of critical revision such as those of Mach or the present work could have been possible without the preceding 
developments and their metaphysical ingredients. Critic, as the negative tool of philosophy, cannot operate in the void but can operate as self-criticism in the process of construction of knowledge.

Open Access This article is distributed under the terms of the Creative Commons Attribution 4.0 International License (http://creativecommons.org/licenses/by/4.0/), which permits unrestricted use, distribution, and reproduction in any medium, provided you give appropriate credit to the original author(s) and the source, provide a link to the Creative Commons license, and indicate if changes were made.

\section{References}

Aristotle. (1994-2010). Physics. The Internet Classics Archive. http://classics.mit.edu/index.html (R. P. Hardie \& R. K. Gaye, Trans.).

Arnold, V., \& Avez, A. (1968). Ergodic problems of classical mechanics. Redwood City, CA: AddisonWesley. (Reprint 1989. Original from 1968 by W. A. Benjamin).

Ballard, K. E. (1960). Leibniz's theory of space and time. Journal of the History of Ideas, 21(1), 49-65.

Bell, J. L. (2002). Time and causation in Gödel's universe. Transcendent Philosophy, 3.

Binkoski, J. P. (2016). On the viability of Galilean relationalism. The British Journal for the Philosophy of Science, 68, 1183-1204.

Burks, A. W. (1946). Peirce's theory of abduction. Philosophy of Science, 1, 301-306.

Carnap, R. (1959). The elimination of metaphysics through logical analysis. In A. J. Ayer (Ed.), Logical Positivism (Chap. 3, pp. 60-81). Glencoe: The Free Press.

Chang, H. (2012). Is water $\mathrm{H}_{2} \mathrm{O}$ ? Evidence, realism and pluralism (Vol. 293)., Boston studies in the philosophy of science Dordrecht: Springer.

Cornu, A. (1876). Détermination de la vitesse de la lumière: d'après des expériences exécutées en 1874 entre l'Observatoire et Montlhéry. Gauthier-Villars

Delhôtel, J. M. (2017). Retaining structure: A relativistic perspective. Journal for General Philosophy of Science, 48(2), 239-256.

Descartes, R. (2015). Discourse on the method of rightly conducting one's reason and of seeking truth in the sciences. Indianapolis: Hackett Publishing Company. (edited by Jonathan Bennett).

Galilei, G. (1914). Dialogs concerning two new sciences. The Macmillan Company (H. Crew, \& A. de Salvio, Trans.)

Goldstein, S., Misra, B., \& Courtage, M. (1981). On intrinsic randomness of dynamical systems. Journal of Statistical Physics, 25(1), 111-126.

Gödel, K. (1949). An example of a new type of cosmological solutions of Einstein's field equations of gravitation. Review of Modern Physics, 21, 447-450.

Hartshorne, C., \& Weiss, P. (Eds.). (1931). Collected papers of Charles Sanders Peirce. Cordele: The Murray Printing Company.

Heelan, P., \& Tymieniecka, A.-T. (Eds.). (2003). Phenomenology world-wide: A guide for research and study (pp. 631-641).

Hegel, G. F. (2001). Science of logic. Blackmask Online. http://www.inkwells.org/index_htm_files/hegel .pdf. Accessed 19 Dec 2017.

Hill, C. O., \& Haddock, G. E. R. (2000). Husserl or Frege? Meaning, objectivity, and mathematics. Chicago: Open Court Publishing.

Hume, D. (1896). Treatise of human nature. Oxford: Clarendon Press. http://oll.libertyfund.org/titles/humea-treatise-of-human-nature.

Husserl, E. (1983). Ideas pertaining to a pure phenomenology and to a phenomenological philosophy. First book: Martinus Nijhoff Publishers (F. Kersten, Trans.).

Husserl, E., \& Lauer, Q. (1956). Philosophy as a strict science. CrossCurrents, 6(4), 325-344. (Original in German by Edmund Husserl, "Philosophie als strenge Wissenschaft," Logos I (1910-11), Tübingen, Mohr, pp. 289-341.).

Kant, I. (1783). What is illustration? Berlinischen Monatsschrift. http://www.columbia.edu/acis/ets/CCREA D/etscc/kant.html (Mary C. Smith, Trans.).

Kant, I. (1787). The critique of pure reason. An electronic classics series publication, (J. M. D. Meiklejohn, Trans.). 
Kant, I. (1798). The conflict of the faculties (the contest of the faculties). Der Streit der Fakultäten. In H. Reiss (Ed.), Kant: Political writings, 2nd ed. (1991). Cambridge: Cambridge University Press. la.utexas.edu/users/hcleaver/330T/350kPEEKantConflictFacNarrow.pdf.

Kant, I. (1993). Grounding for the metaphysics of morals, 3rd edn. Hackett (James W. Ellington, Trans.).

Lissack, M. (2017). Second order science: Examining hidden presuppositions in the practice of science. Foundations of Science, 22(3), 557-573.

Lombardi, O., \& Pérez Ransanz, A. R. (2012). Los Múltiples Mundos de la Ciencia. Un realismo pluralista y su aplicación a la filosofía de la física. Siglo XXI Editores

Mach, E. (1919). The science of mechanics. A critical and historical account of its development. Chicago: The Open Court Publishing Co. (Thomas J. McKormack, Trans.).

Maudlin, T. (1993). Buckets of water and waves of space: Why spacetime is probably a substance. Philosophy of Science, 60(2), 183-203.

McTaggart, J. E. (1908). The unreality of time. Mind, 17(68), 457-474.

Mueller, K. (2014). Towards a general methodology for second-order science. Systemics, Cybernetics and Informatics, 12(5), 33-42.

Newton, I. (1687). Philosophiæ, naturalis principia mathematica ("Mathematical principles of natural philosophy"). London, consulted: Motte translation (1723) published by Daniel Adee publisher (1846). And the Motte translation revised by Florian Cajori (1934) published by Univ of California Press (1999)

Nisbet, R. A. (1971). The degradation of the academic Dogma: The university in America, 1945-1970. The John Dewey Society lectureship series, London

Piaget, J. (1978). El desarrollo de la noción de tiempo en el niño. 04; BF723, P5., S.L. Fondo de cultura económica de España

Piaget, J. (1999). The construction of reality in the Child. Routledge: International Library of Psychology.

Piaget, J., \& García, R. (1989). Psychogenesis and the history of science. New York: Columbia University Press.

Plato. (2014-) Timaeus. Guthemberg project. http://www.gutenberg.org/ebooks/1572. Accessed 16 July 2015.

Poincaré, H. (1913). Science and hypothesis. The foundations of science. The Science Press (George B. Halted, Trans.).

Popper, K. (1959). The Logic of Scientific Discovery (1st ed.). London: Routledge.

Rasch, G. (1968). A mathematical theory of objectivity and its consequences for model construction. In: European meeting on statistics, econometrics and management science, Amsterdam, (Vol. 2).

Russell, B. (1912-1913). On the notion of cause. Proceedings of the Aristotelian Society, 23, 1-26.

Solari, H. G., Natiello, M. A., \& Mindlin, B. G. (1996). Nonlinear dynamics: A two-way trip from physics to math. Bristol: Institute of Physics.

Van Flandern, T. (1998). The speed of gravity-what the experiments say. Physics Letters A, 250(1), 1-11.

Weber, W. (1846). Determinations of electromagnetic meassure; concerning a universal law of electrical action. Prince Jablonowski Society (Leipzig) (pp. 211-378, Trans. by Susan P. Johnson, \& edited by L. Hecht, \& A. K. T. Assis from Wilhelm Weber, "Elektrodynamische Maassbestimmungen: Ueber ein allgemeines Grundgesetz der elektrischen Wirkung," Werke, Vol. III: Galvanismus und Electrodynamik, part 1, edited by H. Weber (Berlin: Julius Springer, 1893) (pp. 25-214).

Wigner, E. P. (1964). Events, laws of nature, and invariance principles. Science, 145(3636), 995-999.

H. G. Solari is professor of physics at the School of Exact and Natural Sciences, University of Buenos Aires and research fellow of the National Research Council of Argentina (CONICET). He has contributed research to physics, mathematics, biology (ecology and epidemiology) and more recently has been engaged in Applied Philosophy of Sciences (defined as a reflexive and critical practice of science grounded in philosophy). Most of his work is of interdisciplinary type.

M. A. Natiello is professor of Applied Mathematics at the Centre for Mathematical Sciences, Lund University. He has contributed research in chemistry, physics, mathematics, biology. His current research interests are Applied Philosophy of Sciences and Population Dynamics with a focus on social, ecological and epidemiological aspects. 